\title{
THE REGULAR REPRESENTATIONS OF MEASURE GROUPOIDS ${ }^{1}$
}

\author{
BY \\ PETER HAHN
}

\begin{abstract}
Techniques are developed to study the regular representation and $\sigma$-regular representations of measure groupoids. Convolution, involution, a modular Hilbert algebra, and local and global versions of the regular representation are defined. The associated von Neumann algebras, each uniquely determined by the groupoid and the cocycle $\sigma$, provide a generalization of the group-measure space construction. When the groupoid is principal and ergodic, these algebras are factors. Necessary and sufficient conditions for the o-regular representations of a principal ergodic groupoid to be of type I, II, or III are given, as well as a description of the flow of weights; these are independent of $\sigma$. To treat nonergodic groupoids, an ergodic decomposition theorem is provided.
\end{abstract}

Introduction. Much of the rich theory associated with locally compact second countable groups can be reformulated entirely in terms of algebra and measure theory. In fact, if an analytic group has a translation-invariant measure class, then the group has a unique locally compact second countable topology for which the given measure class is the Haar measure class [17]. Once a property has been reformulated to remove reference to the topology, it is natural to ask whether that property can be generalized to Mackey's measure groupoids [18]. In [12], we showed that in a suitable sense existence of Haar measure generalizes to groupoids. The present paper is devoted to studying another part of the theory which extends from groups to groupoids-the regular representation. A major tool in this work is the Haar measure.

Although each step in the study is motivated by the group regular representation, the results illuminate an entirely different area. For groupoids arising from the action of a group on a measure space, the commuting algebra of the regular representation is the von Neumann algebra obtained from the group-measure space construction. Using other groupoids, one obtains as special cases other von Neumann algebras introduced to generalize

Presented to the Society, April 25, 1976; received by the editors May 5, 1976 and, in revised form, June 5, 1977.

AMS (MOS) subject classifications (1970). Primary 28A65, 46L10, 46K15.

'Preparation supported by National Science Foundation Grant MPS 74-19876.

- American Mathematical Society 1978 
the group-measure space construction. By treating simultaneously the $\sigma$-regular representations for 2-cocycles $\sigma$ of the groupoid, we obtain as a special case the twisted crossed product of a discrete group acting on an abelian von Neumann algebra as studied by Zeller-Meier [31].

In [12], the modular function $\Delta$ associated with a Haar measure $(\nu, \mu)$ of the groupoid $(G, C)$ was introduced as $\left(d \nu^{-1} / d \nu\right)^{-1}$. It was proved that $\Delta$ is a homomorphism into $\mathbf{R}_{+}^{*}$; changing the Haar measure does not change the similarity class of $\Delta$. In the present paper, $\Delta$ plays a leading role. For the Banach *-algebras, consisting of functions on the groupoid, which are introduced in $\$ 1$, the multiplication is convolution and the involution is given by $f^{*}(x)=\overline{f\left(x^{-1}\right)} \Delta(x)^{-1}$. It is shown in $\$ 2$ that there is a subalgebra of these Banach *-algebras which defines a modular Hilbert algebra. Multiplication by $\Delta$ is the modular operator associated with this modular Hilbert algebra.

The relationship between the local and global representation theories of the groupoid is considered in \$3. Only material needed to study the regular representation is included in this paper. An important tool derived is that the commuting algebra (in the sense of Mackey [19]) of a groupoid representation coincides with the commuting algebra of the algebra representation which it induces. In $\$ 4$ we use the extension $G^{\sigma}$ to extend the several descriptions of the regular representation to the $\sigma$-regular representation as well. The $\sigma$-regular representation of $G$ generates the same von Neumann algebra as a certain subrepresentation of the regular representation of $G^{\circ}$. From this it is easy to construct explicitly a modular Hilbert algebra of functions on $G^{\sigma}$ generating the same von Neumann algebra. Again, $\Delta$ appears as the modular operator. The von Neumann algebra generated by the $\sigma$-regular representation depends only on the measure groupoid $(G, C)$ and the cohomology class of the 2-cocycle $\sigma$.

Principal groupoids generalize free actions of groups; for these groupoids it is easier to analyse the structure of the $\sigma$-regular representation von Neumann algebras than for the general case. We show in $\$ 5$ that the von Neumann algebras of a principal groupoid are factors iff the groupoid is ergodic, semifinite iff $\Delta$ is similar to the identity, type I factors iff the groupoid is essentially transitive. For $\Delta$ nontrivial and the groupoid principal and ergodic, the flow of weights is isomorphic to the closure of the range of $\Delta$ in the sense of [20, \$7]. It is worth emphasizing that these considerations do not depend upon choice of the cocycle $\sigma$, although the von Neumann algebra does.

The purpose of $\S 6$ is to prove a groupoid Ergodic Decomposition Theorem. For principal groupoids the process described effects a central decomposition of the regular representations.

The relationship between the representation theories of similar groupoids $[20, \S 6]$ is not discussed here. A significant contribution in this direction may be found in [7]. 
Notation unexplained in this paper is explained in [12]. Like [12], this paper is based on material contained in the author's doctoral thesis [11]. The acknowledgments made in the introduction to [12] are valid here.

1. Convolution. Just as for groups, one way to introduce the regular representation of measure groupoids is through convolution. Since our generalization of Haar measure is available, convolution for groupoids offers no substantial difficulties; however, the absence of topology makes certain routine verifications technically more complicated. The definition of convolution and an outline of some of its properties have been given by Westman [29], [30], who assumed existence of Haar measure as part of the definition of measure groupoid.

Let $(G, C)$ be a measure groupoid. As defined in [12], a Haar measure $(\nu, \mu)$ for $(G, C)$ consists of a probability measure $\mu \in \tilde{C}$ on $U_{G}$ and a $\sigma$-finite measure $\nu \in C$ on $G$ such that on an i.r. $G \mid U_{0}$ of $G \nu$ has $r$-decomposition $\nu=\int \nu^{u} d \mu(u)$ with respect to $\mu$ satisfying $\left(E \mapsto \int 1_{E}(x y) d \nu^{d(x)}(y)\right)=\nu^{r(x)}$ for all $x \in G \mid U_{0}$. Let $\lambda \in C$ be a symmetric probability measure. Let $\psi=$ $d \mu / d \tilde{\lambda}$. By Remark 3.17 of [12] there is an i.r. $G \mid U$ of $G \mid U_{0}$ and a positive Borel function $P$ on $G \mid U$ such that

(1). If $\lambda^{u}=E \mapsto \int 1_{E}(y) / P(y) d \nu^{u}(y)$ then $\lambda^{u}(G)=\lambda^{u}(G \mid U)=1$ and $\lambda=\int \lambda^{u} d \tilde{\lambda}(u)$ is an $r$-decomposition of $\lambda$ on $G \mid U$.

(2). For $(x, y) \in G^{(2)} \cap(G|U \times G| U), \quad P(x y) P\left(y^{-1} x^{-1}\right)^{-1}=$ $P(x) P(y) P\left(x^{-1}\right)^{-1} P\left(y^{-1}\right)^{-1}$.

Let $\Delta=\left(y \mapsto P(y) \psi \circ r(y) P\left(y^{-1}\right)^{-1} \psi \circ d(y)^{-1}\right)$. The modular function $\Delta$ is a strict homomorphism of $G \mid U$ into the multiplicative group $\mathbf{R}_{+}^{*} ; \nu=$ $\left(E \mapsto \int 1_{E}(y) \psi \circ r(y) P(y) d \lambda(y)\right)$; and for positive Borel $f$ on $G \mid U$, $\int f\left(y^{-1}\right) d \nu(y)=\int f(y) \Delta(y)^{-1} d \nu(y)$. For convenience, in the remainder of this section we will replace $G$ by its inessential reduction $G \mid U$. See Remark 1.9 .

If $f$ is absolutely $\nu$-integrable and $g=f$ a.e., then $\int f d \nu^{u}=\int g d \nu^{u} \mu$-a.e. To assert that $u \mapsto \int f d \nu^{u}$ belongs to $L^{\infty}\left(U_{G}, \tilde{\lambda}\right)$ is thus a property of the $L^{1}(G, \nu)$ class to which $f$ belongs. Let $f^{*}=\left(x \mapsto \overline{f\left(x^{-1}\right)} \Delta(x)^{-1}\right)$. If $f=g$ a.e., then $f^{*}=g^{*}$ a.e.; and

$$
\int\left|f^{*}(x)\right| d \nu(x)=\int\left|f\left(x^{-1}\right)\right| \Delta(x)^{-1} d \nu(x)=\int|f(x)| d \nu(x),
$$

so $\left\|f^{*}\right\|_{1}=\|f\|_{1}, f^{* *}=f,(f+g)^{*}=f^{*}+g^{*},(c f)^{*}=\bar{c} f^{*}$, and $|f|^{*}=\left|f^{*}\right|$.

For $f \in L^{1}(G, \nu)$ and $j, k \in L^{2}\left(U_{G}, \mu\right)$, the extended real number $\int|f(x)||j(d(x)) k(r(x))| d \nu(x)$ depends only on the function classes of $f, j$, and $k$. Let

$$
\|f\|_{I I}=\sup \left\{\int|f(x) j(d(x)) k(r(x))| d \nu(x): \int|j|^{2} d \mu=\int|k|^{2} d \mu=1\right\} .
$$


Let

$$
\|f\|_{I}=\max \left\{\left\|u \mapsto \int|f| d \nu^{u}\right\|_{\infty},\left\|u \mapsto \int\left|f^{*}\right| d \nu^{u}\right\|_{\infty}\right\} .
$$

Let $I I(G, \nu, \mu)=\left\{f \in L^{1}(G, \nu):\|f\|_{I I}<\infty\right\}$ and $I(G, \nu, \mu)=\{f \in$ $\left.L^{1}(G, \nu):\|f\|_{I}<\infty\right\}$. We will abbreviate these sets $I I(G)$ and $I(G)$.

LEMMA 1.1. $\|f\|_{I}=\left\|f^{*}\right\|_{I},\|f\|_{I I}=\left\|f^{*}\right\|,\|f\|_{1} \leqslant\|f\|_{I I} \leqslant\|f\|_{I} \cdot\|\|_{I}$ and \|\|$_{I I}$ are complete norms on $I(G)$ and $I I(G)$, respectively.

Proof. The equalities and inequalities involve only computation. For example,

$$
\begin{aligned}
& \int|f(x) j(d(x)) k(r(x))| d \nu(x) \\
& \quad<\int|f(x)||j(d(x))|^{2} d \nu(x)^{1 / 2} \int|f(x)||k(r(x))|^{2} d \nu(x)^{1 / 2} \\
& \quad=\int\left|f^{*}(x)\right||j(r(x))|^{2} d \nu(x)^{1 / 2} \int|f(x)||k(r(x))|^{2} d \nu(x)^{1 / 2} \\
& \quad=\iint\left|f^{*}(x)\right| d \nu^{u}(x)|j(u)|^{2} d \mu(u)^{1 / 2} \iint|f(x)| d \nu^{u}(x)|k(u)|^{2} d \mu(u)^{1 / 2} \\
& \quad<\left\|u \mapsto \int\left|f^{*}(x)\right| d \nu^{u}(x)\right\|_{\infty}^{1 / 2}\|j\|_{2}\left\|u \mapsto \int|f(x)| d \nu^{u}(x)\right\|_{\infty}^{1 / 2}\|k\|_{2} \\
& \quad<\|f\|_{I}\|j\|_{2}\|k\|_{2},
\end{aligned}
$$

so $\|f\|_{I I} \leqslant\|f\|_{I}$ and if equality holds then $\left\|u \mapsto \int\left|f^{*}\right| d \nu^{u}\right\|_{\infty}=\| u \mapsto$ $\int|f| d \nu^{u} \|_{\infty}$.

It is easy to see that \|\|$_{I}$ and \|\|$_{I I}$ are norms. We show that they are complete norms. Suppose that $\left(f_{n}\right)$ is a \|\|$_{I}$ Cauchy sequence. Then $\left(f_{n}\right)$ is \|\|$_{1}$ Cauchy, so there is $f \in L^{1}(G, \nu)$ such that $\left\|f_{n}-f\right\|_{1} \rightarrow 0$. Hence also $\left\|f_{n}^{*}-f^{*}\right\|_{1} \rightarrow 0$. Since $\iint\left|f_{n}-f\right| d \nu^{u} d \mu(u) \rightarrow 0$, there is a subsequence $\left(f_{n(i)}\right)$ such that $\int\left|f_{n(i)}-f\right| d \nu^{u} \rightarrow 0$ a.e., so $\left|\int\right| f_{n(i)}\left|d \nu^{u}-\int\right| f\left|d \nu^{u}\right|<\int \mid f_{n(i)}-$ $f \mid d \nu^{u} \rightarrow 0$ a.e. Since $\left(f_{n}\right)$ is \|\|$_{I}$ Cauchy, $\left(\left\|f_{n}\right\|_{I}\right)$ is a bounded sequence. Thus $u \mapsto \int|f| d \nu^{u}$ is essentially bounded, as is $u \mapsto \int\left|f^{*}\right| d \nu^{u}$, by the same argument. This proves $f \in I(G)$.

Let $\varepsilon>0$ be given. Choose $n_{0} \in \mathbf{N}$ such that $\left\|f_{n}-f_{m}\right\|_{I}<\varepsilon / 2$ if $n$, $m>n_{0}$. There is a null set $N \subset U_{G}$ such that $\int\left|f_{n}-f_{m}\right| d \nu^{u}<\varepsilon / 2$ if $n$, $m \geqslant n_{0}$ and $u \in U_{G}-N$. For such $u$ and for $n \geqslant n_{0}$ we have

$$
\begin{aligned}
\int\left|f_{n}-f\right| d \nu^{u} & <\int\left|f_{n}-f_{n(i)}\right| d \nu^{u}+\int\left|f_{n(i)}-f\right| d \nu^{u} \\
& <\varepsilon / 2+\int\left|f_{n(i)}-f\right| d \nu^{u} \text { for large } i
\end{aligned}
$$


Since $\int\left|f_{n(i)}-f\right| d \nu^{u} \rightarrow 0$ a.e., we have for a.a. $u$ and for $n \geqslant n_{0} \int \mid f_{n}-$ $f\left|d \nu^{u}<\varepsilon . \int\right| f_{n}^{*}-f^{*} \mid d \nu^{u}$ is treated similarly, showing that $\left\|f_{n}-f\right\|_{I} \rightarrow 0$ and that \|\|$_{I}$ is a complete norm.

To show that \|\|$_{I I}$ is a complete norm, let $\left(f_{n}\right)$ be a \|\|$_{I I}$ Cauchy sequence. As before, there exists $f \in L^{1}(G, \nu)$ such that $\left\|f_{n}-f\right\|_{1} \rightarrow 0$. Also, for each $j$, $k \in L^{2}\left(U_{G}, \mu\right),\left(x \mapsto f_{n}(x) j(d(x)) k(r(x))\right)$ is a Cauchy sequence in $L^{1}(G, v)$. There is a subsequence $\left(f_{n(i)}\right)$ such that $f_{n(i)} \rightarrow f$ a.e. and $f_{n(i)}(j \circ d)(k \circ r)$ converges a.e. This implies that $f_{n(i)}(j \circ d)(k \circ r)$ must converge in $L^{1}$ to $f(j \circ d)(k \circ r)$, so

$$
\begin{aligned}
\int|f(x) j(d(x)) k(r(x))| d \nu(x) & =\lim _{i \rightarrow \infty} \int\left|f_{n(i)}(x) j(d(x)) k(r(x))\right| d \nu(x) \\
& <\sup \left\{\left\|f_{n}(i)\right\|_{I I}: i \in \mathbf{N}\right\}\|j\|_{2}\|k\|_{2} .
\end{aligned}
$$

Thus $f \in I I(G)$.

Suppose $\varepsilon>0$. Choose $m_{0} \in \mathbf{N}$ such that $i>m_{0} \Rightarrow \|(f-$ $\left.f_{n(i)}\right)(j \circ d)(k \circ r) \|_{1}<\varepsilon / 2$. Choose $n_{0}$ such that $m, n \geqslant n_{0} \Rightarrow\left\|f_{n}-f_{m}\right\|_{I I}<$ $\varepsilon / 2$. Then if $n \geqslant n_{0}$

$$
\begin{aligned}
& \int\left|f_{n}(x)-f(x)\right||j(d(x)) k(r(x))| d \nu(x) \\
& \quad<\left\|f_{n}-f_{n(i)}\right\|_{I I}\|j\|_{2}\|k\|_{2}+\left\|\left(f_{n(i)}-f\right)(j \circ d)(k \circ r)\right\|_{1} \\
& <\varepsilon \text { if } i>m_{0}+n_{0} .
\end{aligned}
$$

Thus $\left\|f_{n}-f\right\|_{I I} \rightarrow 0$, so \|\|$_{I I}$ is complete.

REMARK 1.2. If $\mu=\tilde{\lambda}, g \mapsto g P^{-1}$ is a norm-decreasing injection of $L^{\infty}(G, v)$ into $I(G)$.

EXAMPLE 1.3. $G=[0,1] \times[0,1] \subset \mathbf{R}^{2}$ is a principal groupoid if we define $(q, s)(s, t)=(q, t)$ and $(q, s)^{-1}=(s, q)$. Let $m$ be Lebesgue measure on $[0,1]$. $(m \times m, m)$ is a unimodular (symmetric) Haar measure. Let $f(s, t)=$ $1_{[0,1 / 2]}(s)$. $\int f d \delta_{s} \times m=1_{[0,1 / 2]}(s)$ and $\int f^{*} d \delta_{s} \times m=\frac{1}{2}$. The proof of Lemma 1.1 shows that $\|f\|_{I I}<\|f\|_{I}$. Let $g(s, t)=s^{-1 / 4} . g \in I I(G, m \times m, m)$ but $g \notin I(G, m \times m, m)$.

LEMMA 1.4. If $f \in I I(G), k \mapsto\left(u \mapsto \int f(x) k(d(x)) d \nu^{u}(x)\right)$ is a bounded operator $I I_{f}$ on $L^{2}\left(U_{G}, \mu\right)$ and $\|f\|_{I I}=\left\|I I_{|f|}\right\|$. If $f \in I(G)$ then $k \mapsto\left(u \mapsto \int f(x) k(d(x)) d \nu^{u}(x)\right)$ and $k \mapsto\left(u \mapsto \int f(x) k(r(x)) d \nu^{u}(x)\right)$ are bounded operators $I_{f}$ and $I_{f}^{\prime}$ on $L^{1}\left(U_{G}, \mu\right)$ and $\|f\|_{I}=\max \left\{\left\|I_{|f|}\right\|,\left\|I_{|f|}^{\prime}\right\|\right\}$.

Proof. We prove the statements about $I(G)$. 


$$
\begin{gathered}
\int\left|\int f(x) k(d(x)) d \nu^{u}(x)\right| d \mu(u) \\
\leqslant \int|f(x) k(d(x))| d \nu(x)=\int\left|f^{*}(x)\right||k(r(x))| d \nu(x) \\
=\iint\left|f^{*}(x)\right| d \nu^{u}(x)|k(u)| d \mu(u) \leqslant\left\|u \mapsto \int\left|f^{*}\right| d \nu^{u}\right\|_{\infty}\|k\|_{1}
\end{gathered}
$$

and $\int\left|\int f(x) k(r(x)) d \nu^{u}(x)\right| d \mu(u) \leqslant\left\|u \mapsto \int|f| d \nu^{u}\right\|_{\infty}\|k\|_{1}$. Thus $I_{f}^{\prime}$ and $I_{f}$ are bounded on $L^{1}\left(U_{G}, \mu\right)$ by $\|f\|_{I}$. Equality of norms holds for $|f|$ because on any $\sigma$-finite measure space $\|g\|_{\infty}=\sup \left\{\int|g k|:\|k\|_{1}=1\right\}$.

Now we accumulate the facts necessary to introduce the convolution product on $I I(G)$. In these calculations, as in others that follow, we appeal to Tonelli's Theorem or to Fubini's Theorem in order to justify a change in the order of integration within a fibered product. Suppose $f, g \in I I(G)$ and $j$, $k \in L^{2}\left(U_{G}, \mu\right)$. If $f, g, j, k \geqslant 0$, then Tonelli's Theorem justifies the following calculation:

$$
\begin{aligned}
\left(I I_{f} I_{g} j, k\right) & =\int f(y) \int g(x) j(d(x)) d \nu^{d(y)}(x) \overline{k(r(y))} d \nu(y) \\
= & \iiint f(y) g\left(y^{-1} x\right) j(d(x)) \overline{k(r(y))} d \nu^{u}(x) d \nu^{u}(y) d \mu(u) \\
= & \iiint f(y) g\left(y^{-1} x\right) j(d(x)) \overline{k(u)} d \nu^{u}(y) d \nu^{u}(x) d \mu(u) \\
= & \iiint f(x y) g\left(y^{-1}\right) d \nu^{d(x)}(y) j(d(x)) d \nu^{u}(x) \overline{k(u)} d \mu(u) .
\end{aligned}
$$

Because

$$
\begin{aligned}
\iiint\left|f(y) g\left(y^{-1} x\right) j(d(x)) k(r(y))\right| d \nu^{u}(x) d \nu^{u}(y) d \mu(u) \\
=\left(I I_{|J|} I I_{|g|}|j|,|k|\right) \leqslant\|f\|_{I I}\|g\|_{I I}\|j\|_{2}\|k\|_{2}
\end{aligned}
$$

calculation (1.5) is valid for complex-valued $f, g, j, k$ by Fubini's Theorem. Setting $j=k=1$, we find that the Borel function

$$
x \mapsto \int f(x y) g\left(y^{-1}\right) d \nu^{d(x)}(y)
$$

is an element of $L^{1}(G, \nu)$ and equals $\int f(y) g\left(y^{-1} x\right) d \nu^{r(x)}(y) \nu$-a.e.

If $f_{1}=f$ a.e. and $g_{1}=g$ a.e., then

$$
\begin{gathered}
\int\left|\int f(x y) g\left(y^{-1}\right) d \nu^{d(x)}(y)-\int f_{1}(x y) g_{1}\left(y^{-1}\right) d \nu^{d(x)}(y)\right| d \nu(x) \\
\leqslant \iint|f(x y)|\left|g\left(y^{-1}\right)-g_{1}\left(y^{-1}\right)\right| d \nu^{d(x)}(y) d \nu(x) \\
\quad+\iint\left|f_{1}(x y)-f(x y)\right|\left|g_{1}\left(y^{-1}\right)\right| d \nu^{d(x)}(y) d \nu(x) \\
=\left(I I_{|f|} I I_{\left|g-g_{1}\right|} 1,1\right)+\left(I I_{\mid f_{1}-\Omega} I I_{\left|g_{1}\right|} 1,1\right)=0
\end{gathered}
$$


Thus, if we define $f * g=\left(x \mapsto \int f(x y) g\left(y^{-1}\right) d \nu^{d(x)}(y)\right)$, then the convolution $(f, g) \mapsto f * g$ is a well-defined function from $I I(G) \times I I(G)$ into $L^{1}(G, \nu)$. Using the fact that $\Delta$ is a strict homomorphism, we calculate that for $\nu$-a.e. $x$

$$
\begin{aligned}
g^{*} * f^{*}(x) & =\int g^{*}(x y) f^{*}\left(y^{-1}\right) d \nu^{d(x)}(y) \\
& =\int \overline{g\left(y^{-1} x^{-1}\right)} \Delta(x y)^{-1} \overline{f(y)} \Delta(y) d \nu^{d(x)}(y) \\
& =\Delta(x)^{-1}\left(\int g\left(y^{-1}\right) f\left(x^{-1} y\right) d \nu^{d\left(x^{-1}\right)}(y)\right) \\
& =\Delta(x)^{-1} \overline{f * g\left(x^{-1}\right)}=(f * g)^{*}(x),
\end{aligned}
$$

so $(f * g)^{*}=g^{*} * f^{*}$ in $L^{1}(G, \nu)$.

Calculation (1.5) and Lemma 1.4 show that

$$
\begin{aligned}
\int|f * g(x) j(d(x)) \overline{k(r(x))}| d \nu(x) & \leqslant\left\|I I_{|f|}\right\|\left\|I I_{|g|}\right\|\|j\|_{2}\|k\|_{2} \\
& =\|f\|_{I I}\|g\|_{I I}\|j\|_{2}\|k\|_{2},
\end{aligned}
$$

so $f * g \in I I(G)$ and $\|f * g\|_{I I} \leqslant\|f\|_{I I}\|g\|_{I I}$. With $j \in L^{1}\left(U_{G}, \mu\right)$ and $k=$ 1 , applying calculation (1.5) to $f, g \in I(G)$ shows

$$
\begin{aligned}
\int\left|\int\right| f * g\left|(x) j(d(x)) d \nu^{u}(x)\right| d \mu(u) & \leqslant\left\|I_{|f|} I_{|g|}|j|\right\|_{1} \\
& \leqslant\left\|I_{|f|}\right\|\left\|I_{|g|}\right\|\|j\|_{1} \leqslant\|f\|_{I}\|g\|_{I}\|j\|_{1} .
\end{aligned}
$$

By Lemma 1.4, $f * g \in I(G)$ and $\|f * g\|_{I} \leqslant\|f\|_{I}\|g\|_{I}$. We have proved

LEMMA 1.6. $I(G)$ and $I I(G)$ are closed under convolution $(f, g) \mapsto f * g$. $\|f * g\|_{I} \leqslant\|f\|_{I}\|g\|_{I},\|f * g\|_{I I} \leqslant\|f\|_{I I}\|g\|_{I I}$, and $(f * g)^{*}=g^{*} * f^{*}$ a.e.

LEMMA 1.7. $f \mapsto f * g$ and $g \mapsto f * g$ are linear; $f *(g * h)=(f * g) * h$ for $f, g, h \in I I(G)$.

Proof. Linearity is obvious. Associativity is proved as it is for groups. If $f$, $g, h \in I I(G)$ are nonnegative,

$$
\begin{aligned}
f *(g * h)(x) & =\int f(x y)(g * h)\left(y^{-1}\right) d \nu^{d(x)}(y) \\
& =\iint f(x y) g\left(y^{-1} z\right) h\left(z^{-1}\right) d \nu^{r(y)}(z) d \nu^{d(x)}(y) \\
& =\iint f(x y) g\left(y^{-1} z\right) h\left(z^{-1}\right) d \nu^{d(x)}(y) d \nu^{d(x)}(z) \\
& =\iint f(x y) g\left(y^{-1} z\right) h\left(z^{-1}\right) d \nu^{r(z)}(y) d \nu^{d(x)}(z)
\end{aligned}
$$




$$
\begin{aligned}
& =\iint f(x z y) g\left(y^{-1}\right) h\left(z^{-1}\right) d \nu^{d(z)}(y) d \nu^{d(x)}(z) \\
& =\int f * g(x z) h\left(z^{-1}\right) d \nu^{d(x)}(z) \\
& =(f * g) * h(x) .
\end{aligned}
$$

For $f, g, h \in I I(G)$ arbitrary, write each as a linear combination of nonnegative elements of $I I(G)$ and expand by linearity.

The foregoing results combine to prove

TheOREM 1.8. $I(G, \nu, \mu)$ and $I I(G, \nu, \mu)$ are Banach *-algebras.

REMARK 1.9. $I I(G)$ is the groupoid algebra of Westman [30]. The elements and operations of both algebras $I(G)$ and $I I(G)$ are unchanged by modification on null sets, so $I(G)$ and $I I(G)$ may be regarded as algebras associated with any i.r. of $G$ or with any groupoid of which $G$ is an i.r. Moreover, if $(\nu, \mu)$ and $\left(\nu_{1}, \mu\right)$ are Haar measures, then $I(G, \nu, \mu)$ and $I\left(G, \nu_{1}, \mu\right)$ are isomorphic. Indeed, it follows from Theorem 3.9 of [12] that there is a positive Borel function $\phi$ on $U_{G}$ such that $\phi \circ d=d \nu_{1} / d \nu . f \mapsto f(\phi \circ d)$ is an isomorphism of $I\left(G, \nu_{1}, \mu\right)$ onto $I(G, \nu, \mu)$.

REMARK 1.10. Professor Mackey has suggested that convolution of functions on groupoids can be interpreted in terms of convolution of measures absolutely continuous with respect to $\lambda$. Assume $\mu=\tilde{\lambda}, P=d \nu / d \lambda$. For a complex measure $\alpha$ on $G$, let $\alpha^{*}$ be the measure $E \mapsto \alpha\left(E^{-1}\right)^{-}$. If $\alpha \ll \lambda$, let $\alpha^{u}=E \mapsto \int_{E}(d \alpha / d \lambda) d \lambda^{u}$ and let $\left\|\alpha^{u}\right\|$ be the total variation of $\alpha^{u}$. If $u \mapsto\left\|\alpha^{u}\right\|$ and $u \rightarrow\left\|\left(\alpha^{-1}\right)^{u}\right\|$ belong to $L^{\infty}\left(U_{G}, \tilde{\lambda}\right)$ then $P^{-1}(d \alpha / d \lambda) \in$ $I(G, \nu, \tilde{\lambda})$; and if $\alpha$ and $\beta$ are two such measures then their convolution is defined by $\alpha * \beta(E)=\int \beta^{d(y)}\left(y^{-1} E\right) d \alpha(y)$. It is easily verified that if $f \in$ $I(G, \nu, \tilde{\lambda})$, then $\alpha_{f}(E)=\int_{E} f d \lambda$ defines one of these measures, $\alpha_{\left(f^{*}\right)}=\left(\alpha_{f}\right)^{*}$, and $\alpha_{(f * g)}=\alpha_{f} * \alpha_{g}$.

Associativity can be demonstrated directly. Since

$$
\begin{aligned}
& \int f d(\alpha * \beta)^{u}=\iint f(x y) d \beta^{d(x)}(y) d \alpha^{u}(x) \\
&(\alpha * \beta) * \gamma(E)=\int \gamma^{d(x)}\left(x^{-1} E\right) d(\alpha * \beta)(x) \\
&=\iint 1_{E}(x z) d \gamma^{d(x)}(z) d(\alpha * \beta)(x) \\
&=\iiint 1_{E}(x y z) d \gamma^{d(x y)}(z) d \beta^{d(x)}(y) d \alpha(x) \\
&=\iint(\beta * \gamma)^{d(x)}\left(x^{-1} E\right) d \alpha(x)=\alpha *(\beta * \gamma)(E) .
\end{aligned}
$$

When $G$ is principal, the measures $\alpha^{u}$ may be transferred to $U_{G}$ from 
$\{u\} \times U_{G}$. With this convention convolution takes the following form, suggestive of Markov kernels:

$$
(\alpha * \beta)^{u}(E)=\int \beta^{v}(E) d \alpha^{u}(v) .
$$

EXAMPLE 1.11. As in Example 3.16 of [12], let $\mathrm{g}$ with Haar measure $h$ act on $(S, \mu)$, so that $(\mu \times h, \mu)$ is a Haar measure for the measure groupoid $(S \times g$, $[\mu \times h])$ and the modular functions $\Delta$ of $S \times \mathfrak{g}$ and $\delta$ of g satisfy $d(\mu$. $\left.x^{-1}\right) / d \mu=\delta(x) \Delta(s, x)^{-1}$ for $h$-a.a. $x$. The convolution is

$$
f * g(s, x)=\int f(s, x y) g\left(s x y, y^{-1}\right) d h(y)=\int f(s, y) g\left(s y, y^{-1} x\right) d h(y) .
$$

It agrees with the convolution used by Glimm [10]. The norm $\|f\|_{I I}$ is

$$
\begin{aligned}
& \sup \left\{\int|f(s, x) j(s x) k(s)| d \mu \times h(s, x):\right. \\
& \left.\int|j(s)|^{2} d \mu(s)=\int|k(s)|^{2} d \mu(s)=1\right\} .
\end{aligned}
$$

The norm $\|f\|_{I}$ is

$$
\begin{aligned}
& \max \left\{\left\|s \mapsto \int|f(s, x)| d h(x)\right\|_{\infty}\right. \\
&\left.\left\|s \mapsto \int\left|f\left(s x, x^{-1}\right)\right| \Delta\left(s x, x^{-1}\right) d h(x)\right\|_{\infty}\right\} .
\end{aligned}
$$

The involution is $f^{*}(s, x)=\overline{f\left(s x, x^{-1}\right)} \Delta\left(s x, x^{-1}\right)$.

Let $L_{c}^{\infty}$ be the subspace of $L^{\infty}(S \times \mathfrak{g}, \mu \times h)$ of functions $f$ vanishing a.e. outside of a set $S \times K_{f}, K_{f} \subset g$ compact. For $f, g \in L_{c}^{\infty}$, let $f^{\circ}(s, x)=\overline{f\left(s x, x^{-1}\right)} \delta(x)^{-1}$ and $f * g(s, x)=\int f(s, x y) g\left(s x y, y^{-1}\right) d h(y)$. $\|f * g\|<\|f\|_{\infty}\|g\|_{\infty} h\left(K_{g}\right)$ and $f * g$ vanishes a.e. off $S \times\left(K_{f} K_{g}\right)$. Arguing as in the proof of Theorem 1.8, one can prove that $L_{c}^{\infty}$ is a ${ }^{*}$-algebra.

For $f \in L_{c}^{\infty}$ define $\Phi f$ by $\Phi f(s, x)=f(s, x) \Delta(s, x)^{-1 / 2} \delta(x)^{1 / 2} . \Phi$ is a *-homomorphism of $L_{c}^{\infty}$ into $I I(S \times g)$. By restricting $\Phi$ to the continuous functions on $\mathrm{g}$ with compact support and then extending to $L^{1}(\mathrm{~g}, h)$, we obtain a norm-decreasing injective *-homomorphism $\Phi$ of $L^{1}(g, h)$ into $I I(S$ $\times \mathrm{g})$. If $S=\{e\}$ then $L^{1}(\mathrm{~g}, h)=I(S \times \mathrm{g})=I I(S \times \mathrm{g})$.

2. The modular Hilbert algebra. Let $(\nu, \mu)$ be a Haar measure for the measure groupoid $(G, C)$. Let $U, \psi, P$, and $\lambda=\int \lambda^{u} d \tilde{\lambda}(u)$ be as at the beginning of $\S 1$. We are interested in a particular representation of $I I(G, \nu, \mu)=I I(G)$ on the Hilbert space $L^{2}(G, \nu)$.

LEMMA 2.1. $L_{f}(j)=\left(x \mapsto \int f(x y) j\left(y^{-1}\right) d \nu^{d(x)}(y)\right)$ defines a norm-decreasing *-representation $L$ of $I I(G)$ on $L^{2}(G, v)$, called the left regular representation. 
Proof. Suppose $f \in I I(G), j, k \in L^{2}(G, \nu)$, and $f, j, k \geqslant 0$.

$$
\begin{aligned}
\iint f(x y) j\left(y^{-1}\right) d \nu^{d(x)}(y) k(x) d \nu(x) \\
\quad=\int_{U} \int_{G \mid U} \int_{G \mid U} f(y) j\left(y^{-1} x\right) k(x) d \nu^{u}(y) d \nu^{u}(x) d \mu(u) \\
\quad \leqslant \int_{G \mid U} f(y)\left(\int_{G \mid U} j(x)^{2} d \nu^{d(y)}(x)\right)^{1 / 2}\left(\int_{G \mid U} k(x)^{2} d \nu^{r(y)}(x)\right)^{1 / 2} d \nu(y) \\
\leqslant\|f\|_{I I}\left\|u \mapsto \int j(x)^{2} d \nu^{u}(x)^{1 / 2}\right\|\left\|_{2}\right\| u \mapsto \int k(x) d \nu^{u}(x)^{1 / 2} \|_{2} \\
\quad=\|f\|_{I I}\|j\|_{2}\|k\|_{2} .
\end{aligned}
$$

Thus $L$ is a norm-decreasing *-representation.

COROLLARY 2.2. The restriction of $L$ to $I(G)$ is a norm-decreasing *-representation.

We will show that $\left\{L_{f}: f \in I(G)\right\}$ is nondegenerate. The von Neumann algebra generated by this *-algebra of operators on $L^{2}(G, \nu)$ is the principal object of study in this paper. The main result of this section is a description of a modular Hilbert algebra generating $\left\{L_{f}: f \in I(G)\right\}^{\prime \prime}$.

As in $\S 1$, we will pass to the inessential reduction $G \mid U$. Again, this is simply for convenience.

Definition 2.3. An absolutely integrable Borel function $f$ is strictly $\Delta_{a^{-}}$ bounded if for all $x f(x)=0$ or $\Delta(x) \in[1 / a, a]$. An absolutely integrable Borel function $g$ is $\Delta_{a}$-bounded if $g$ agrees a.e. with a strictly $\Delta_{a}$-bounded function. An a.e. function class is $\Delta_{a}$-bounded if it contains a $\Delta_{a}$-bounded member. A function or function class is $\Delta$-bounded if it is $\Delta_{a}$-bounded for some $a \geqslant 1$.

LEMMA 2.4. $\{f \in I I(G): f$ is $\Delta$-bounded $\}$ is an involutive subalgebra of $I I(G)$.

Proof. Suppose $f_{0}$ is strictly $\Delta_{a}$-bounded and $g_{0}$ is strictly $\Delta_{b}$-bounded. Since $\Delta(x)=\Delta(x y) \Delta(y)^{-1}$, if $\Delta(x)>a b$ then either $\Delta(x y)>a$ or $\Delta\left(y^{-1}\right)>$ $b$. Thus if $\Delta(x)>a b$ then $f_{0}(x y) g_{0}\left(y^{-1}\right)=0$ for all $y \in r^{-1}(d(x))$, so $f_{0} * g_{0}(x)=\int f_{0}(x y) g_{0}\left(y^{-1}\right) d \nu^{d(x)}(y)=0$. Similarly, if $\Delta(x)<1 /(a b)$, then $f_{0} * g_{0}(x)=0$. Thus $f_{0} * g_{0}$ is strictly $\Delta_{a b}$-bounded; and this implies that the convolution of two $\Delta$-bounded elements of $I I(G)$ is $\Delta$-bounded.

If $f_{0}$ is strictly $\Delta_{a}$-bounded, then $\left\{x: f_{0}^{*}(x) \neq 0\right\}=\left\{x: f_{0}(x) \neq 0\right\}^{-1} \subset\{x$ : $1 / a \leqslant \Delta(x) \leqslant a\}^{-1}=\left\{x: 1 / a \leqslant \Delta\left(x^{-1}\right) \leqslant a\right\}=\{x: 1 / a \leqslant \Delta(x) \leqslant a\}$, so $f_{0}^{*}$ is $\Delta_{a}$-bounded. Thus the involute of a $\Delta$-bounded element of $I I(G)$ is $\Delta$-bounded. Clearly, $f \mapsto c f$ and $(f, g) \mapsto f+g$ preserve $\Delta$-boundedness. 
Definition 2.5. $\mathscr{U}_{I I}=L^{2}(G, v) \cap\{f \in I I(G): f$ is $\Delta$-bounded $\}$. $\mathscr{Q}_{I}=$ थ $_{I I} \cap I(G)$.

LEMMA 2.6. Let $E(a)=\left\{y \in G: 1 / a \leqslant P(y) \leqslant a, 1 / a \leqslant P\left(y^{-1}\right) \leqslant a\right.$, $1 / a \leqslant \psi(r(y)) \leqslant a$, and $1 / a \leqslant \psi(d(y)) \leqslant a\}$. If $f=f 1_{E(a)}$ a.e. for some $a \geqslant 1$ and $f \in L^{\infty}(G, \nu)$, then $f \in \mathcal{U}_{I}$.

Proof. By enlarging $a$ and modifying $f$ on a null Borel set, we may assume $f=f 1_{E(a)}$ and $|f| \leqslant a . \int|f|^{2} d \nu=\int|f|^{2}(\psi \circ r) P d \lambda \leqslant a^{4}$ since $\lambda(G)=1$. Thus $f \in L^{2}(G, \nu) . \int|f| d \nu^{u}=\int|f| P d \lambda^{u} \leqslant a^{2}$ since $\lambda^{u}(G)=1$.

$$
\begin{aligned}
\int\left|f^{*}\right| d \nu^{u} & =\int\left|f\left(x^{-1}\right)\right| P\left(x^{-1}\right) \psi(d(x)) P(x)^{-1} \psi(r(x))^{-1} d \nu^{u}(x) \\
& =\int\left|f\left(x^{-1}\right)\right| P\left(x^{-1}\right) \psi(d(x)) \psi(r(x))^{-1} d \lambda^{u}(x) \leqslant a^{4} .
\end{aligned}
$$

Thus $f \in I(G)$. Finally, we show $f$ is strictly $\Delta$-bounded. Suppose $f(y) \neq 0$. Then $y \in E(a)$, so $\Delta(y)=P(y) \psi(r(y)) P\left(y^{-1}\right) \psi(d(y))^{-1} \in\left[a^{-4}, a^{4}\right]$.

Corollary 2.7. $\bigcup_{I} \cap L^{\infty}(G, \nu)$ is dense in $L^{2}(G, \nu)$.

Proof. Let $g \in L^{2}(G, \nu)$; let $g_{n}(y)=g(y)$ if $|g(y)| \leqslant n, g_{n}(y)=0$ otherwise. $g_{n} 1_{E(n)} \in \mathcal{U}_{I} \cap L^{\infty}(G, \nu)$. Since $E(n) \subset E(n+1)$ and $\cup E(n)=G$, $\left|g-g_{n} 1_{E(n)}\right|^{2}$ converges pointwise to zero, dominated by $|g|^{2}$, so the Dominated Convergence Theorem implies $\left\|g-g_{n} 1_{E(n)}\right\|_{2} \rightarrow 0$.

LEMMA 2.8. $\mathcal{U}_{I} \subset I(G)$ and $\vartheta_{I I} \subset I I(G)$ are nonempty complex involutive subalgebras.

Proof. We show $\mathscr{U}_{I}$ and $\mathscr{U}_{I I}$ are closed under convolution and involution. If $f \in L^{2}(G, \nu)$ is $\Delta_{a}$-bounded, then

$$
\begin{aligned}
\int\left|f^{*}\right|^{2} d \nu & =\int\left|f\left(y^{-1}\right)\right|^{2} \Delta\left(y^{-1}\right)^{2} d \nu(y) \\
& =\int|f(y)|^{2} \Delta(y) d \nu(y) \leqslant a\|f\|_{2}^{2}
\end{aligned}
$$

By Lemma 2.1, $\|f * g\|_{2}=\left\|L_{f}(g)\right\|_{2} \leqslant\|f\|_{I I}\|g\|_{2}$ if $f, g \in \mathcal{U}_{I I}$. By Lemma 2.4, if $f$ and $g$ are $\Delta$-bounded, so are $f * g$ and $f^{*}$.

LEMMA 2.9. If $h \in L^{2}(G, \nu) \cap I(G)$ is such that $(\phi * \psi, h)=0$ for all $\phi$, $\psi \in \mathcal{U}_{I} \cap L^{\infty}(G, \nu)$, then $h=0$ a.e.

Proof. Let $E(n)$ be as in Lemma 2.6, $F(1), F(2), \ldots$ a generating algebra for the Borel sets $\mathscr{B}(G)$ of $G$. Let $\phi_{n, m}=1_{E(n)} 1_{F(m)}$ and $\phi_{n, m}^{\prime}=\phi_{n, m} / P$. By Lemma 2.6, $\phi_{n, m}$ and $\phi_{n, m}^{\prime}$ belong to $\mathcal{U}_{I} \cap L^{\infty}(G, v)$. By Fubini's Theorem,

$$
\begin{aligned}
0 & =\left(\phi_{n, m}^{\prime} * \phi_{p, q}, h\right) \\
& =\iint \phi_{n, m}(y) \int \phi_{p, q}\left(y^{-1} x\right) \overline{h(x)} d \nu^{r(y)}(x) d \lambda^{u}(y) d \mu(u) .
\end{aligned}
$$


Since $h \in I(G), \iiint \phi_{p, q}\left(y^{-1} x\right)|h(x)| d \nu^{u}(x) d \lambda^{u}(y) d \mu(u) \leqslant\|h\|_{I}$. By the Dominated Convergence Theorem, $\iint_{F(m)} \int \phi_{p, q}\left(y^{-1} x\right) \overline{h(x)} d \nu^{r(y)}(y) d \lambda^{u}$ (y) $d \mu(u)=0$. By the Caratheodory Extension Theorem, there is a null Borel set $N$ such that $y \in G-N$ and $(p, q) \in \mathbf{N} \times \mathbf{N}$ imply $\int \phi_{p, q}\left(y^{-1} x\right) \overline{h(x)} d \nu^{r(y)}(x)=0$. By the Dominated Convergence Theorem again, there is a null Borel set $N_{1}$ such that if $(m, y) \in \mathbf{N} \times\left(G-N_{1}\right)$, $\int 1_{F(m)}\left(y^{-1} x\right) \overline{h(x)} d \nu^{r(y)}(x)=0$ and $\int|h(x)| d \nu^{r(y)}(x) \leqslant\|h\|_{I}$. Thus if $(m, y)$ $\in \mathbf{N} \times\left(G-N_{1}\right), \quad \int 1_{F(m)}(x) \overline{h(y x)} d \nu^{d(y)}(x)=0$. Hence if $y \in N_{1}$, $(x \mapsto h(y x))=0 \quad \nu^{d(y)}$-a.e., so $\iint|h(y x)| d \lambda^{d(y)}(x) d \lambda(y)=0$, so $h=0$ a.e.

This lemma can also be obtained from results in $\$ 3$.

On $\mathscr{Q}_{I}$ we define a complex one-parameter group of automorphisms $f \mapsto \Delta^{z} f$ for $z \in \mathbf{C}$. Indeed, if $f \in \mathcal{Q}_{I}$ is $\Delta_{a}$-bounded, so is $\Delta^{z} f$, and $\left\|\Delta^{z} f\right\|_{2} \leqslant$ $a^{|\operatorname{Rez} z|}\|f\|_{2}$ and $\left\|\Delta^{z} f\right\|_{I} \leqslant a^{|\operatorname{Rez} z|}\|f\|_{I}$.

We verify

$$
\begin{gathered}
\left(\Delta^{z} f\right)^{*}(y)=\left(\Delta^{z}\left(y^{-1}\right) f\left(y^{-1}\right)\right)-\Delta\left(y^{-1}\right)=\left(\Delta^{-\bar{z}} f^{*}\right)(y), \\
\left(\Delta^{z} f, g\right)=\int \Delta^{z}(y) f(y) \overline{g(y)} d \nu(y)=\left(f, \Delta^{\bar{z}} g\right)
\end{gathered}
$$

and

$$
\begin{aligned}
\left(\Delta^{1} f^{*}, g^{*}\right) & =\int \Delta(y) \overline{f\left(y^{-1}\right)} \Delta(y)^{-1} g\left(y^{-1}\right) \Delta(y)^{-1} d \nu(y) \\
& =\int g(y) \overline{f(y)} d \nu(y)=(g, f) .
\end{aligned}
$$

LEMMA 2.13. If $f, g \in \mathcal{Q}_{I}$ then $z \mapsto\left(\Delta^{2} f, g\right)$ is an entire function.

Proof. Since $f$ and $g$ are $\Delta$-bounded, this follows from standard complex analysis (e.g. use Theorem V.6.1 of [13]).

Lemma 2.14. Let $t \in \mathbf{R}$. If $g \in U_{I}$ is such that $\left(\left(1+\Delta^{t}\right) f, g\right)=0$ for all $f \in \mathcal{U}_{1}$, then $g=0$ a.e.

Proof. $\left(1+\Delta^{t}(y)\right)>0$ for all $y \in G$. If $\int\left(1+\Delta^{t}(y)\right) g(y) \overline{g(y)} d \nu(y)=0$, $\|g\|_{2}=0$.

The definition of modular Hilbert algebra is given in [27]. We use * for involution instead of the "sharp" symbol used in [27].

THEOREM 2.15. $Q_{I}$ is a modular Hilbert algebra dense in $L^{2}(G, \nu)$. The left von Neumann algebra $L\left(\mathcal{U}_{I}\right)^{\prime \prime}$ of $\mathcal{U}_{I}$ coincides with the weak closures of $L(I(G))$ and $L(I I(G)) . L\left(U_{I}\right)^{\prime \prime}=L(I(G))^{\prime \prime}=L(I I(G))^{\prime \prime}$.

Proof. In the definition of modular Hilbert algebra, Property $I$ and Property II follow from $(f * g, h)=\left(L_{f} g, h\right)=\left(g, L_{f} * h\right)=\left(g, f^{*} * h\right)$ and 
$\|g \mapsto f * g\|=\left\|L_{f}\right\| \leqslant\|f\|_{I}$ (Corollary 2.1). Property III is an immediate consequence of Lemma 2.9. Property IV is (2.10), Property V is (2.11), and Property VI is (2.12). Property VII is Lemma 2.13, and Property VIII is Lemma 2.14.

By Corollary 2.7, $\vartheta_{I}$ is dense in $L^{2}(G, v)$. Since $L\left(U_{I}\right) \subset L(I(G)) \subset$ $L(I I(G))$ and the weak closure of the *-algebra $L\left(U_{I}\right)$ contains the identity, it suffices to show that the weak closure of $L\left(U_{I}\right)$ contains $L(I I(G))$. For $g \in I I(G)$ we adopt notation from the proof of Corollary 2.7. Let $f_{n}=$ $g_{n} 1_{E(n)} \in \mathcal{U}_{1}$. If $j, k \in L^{2}(G, v),\left(L\left(g-f_{n}\right) j, k\right) \rightarrow 0$ by the Dominated Convergence Theorem, because

$$
\begin{aligned}
\int\left|g(x y)-f_{n}(x y)\right|\left|j\left(y^{-1}\right) k(x)\right| d \nu^{d(x)}(y) d \nu(x) \\
=\left(L_{\left|g-f_{n}\right|}|j|,|k|\right)<\|g\|_{I I}\|j\|_{2}\|k\|_{2} .
\end{aligned}
$$

Theorem 2.15 provides all of the power of the Tomita-Takesaki theory for study of the von Neumann algebra $L(I(G))^{\prime \prime}$. The modular involution $J$ is

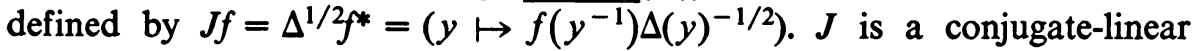
isometry of $L^{2}(G, \nu)$ with itself; and $S \mapsto J S J$ is a conjugate-isomorphism of $L(I(G))^{\prime \prime}$ with its commutant. More will be said about conjugateisomorphisms in $\S 5$.

The notation used in the present section tends to suppress the fact that $\mathscr{Q}_{I}$ depends upon the choice of the Haar measure $(\nu, \mu)$. It appears that $L\left(U_{I}\right)^{\prime \prime}$ also depends upon the choice of Haar measure, but this is not the case. In the next section we provide an alternate description of $L\left(\mathcal{U}_{I}\right)^{\prime}$, one which will be proved to be independent of the Haar measure.

3. Inducing representations to the groupoid algebras. Let $(G, C)$ be a measure groupoid. By a representation of $(G, C)$ on a separable Hilbert space $H$ we mean a homomorphism of $(G, C)$ into the unitary group, endowed with the weak Borel structure [19]. If $X$ is a representation of $\left(G,\left[s \lambda^{u} d \mu(u)\right]\right)$, Mackey has defined the commuting algebra $X^{\prime}$ of $X$ to be the subalgebra of the decomposable operators on $L^{2}\left(U_{G}, \tilde{\lambda}, H\right)$ consisting of operators $T=$ $\int T(u) d \tilde{\lambda}(u)$ such that $T(r(x)) X(x)=X(x) T(d(x))$ for $\lambda$-a.a. $x$ [19].

Since every group representation gives rise to a representation of the group algebra by integration with respect to the Haar measure, one hopes that this relationship can be generalized to measure groupoids. The appropriate formula $\left(X_{f} j, k\right)=\int f(x)(X(x) j(d(x)), k(r(x))) d \nu(x)$ has been given by Westman in an unpublished paper [30]. In this section we give details of this "inducing" construction and show that $X^{\prime}$ is the commutant of $\left\{X_{f}: f \in\right.$ $I I(G)\}$. In particular, the regular representation defined and studied in $\$ 2$ is unitarily equivalent to a representation of $I I(G)$ induced by a representation of $G$, provided $G$ is ergodic. 
Let $(\nu, \mu)$ be a Haar measure for $(G, C)$; let $U, \psi, P$, and $\lambda=\int \lambda^{u} d \tilde{\lambda}(u)$ be as at the beginning of $\S 1$. As before, we assume for convenience that $G=G \mid U$.

LEMMA 3.1. Let $H$ be a separable Hilbert space, $\mathcal{L}(H)$ its algebra of bounded operators. Let $X: G \rightarrow \mathcal{L}(H)$ be a function such that $x \mapsto(X(x) j, k)$ is Borel for all $j, k \in H$ and $\|X(x)\|<1$ a.e. For $f \in I I(G),\left(X_{f} j, k\right)=$ $\int f(x)(X(x) j(d(x)), k(r(x))) d v(x)$ defines an operator $X_{f}$ on the Hilbert space $L^{2}\left(U_{G}, \mu, H\right)$ and $\left\|X_{f}\right\| \leqslant\|f\|_{I I}$.

Proof. Since $L^{2}\left(U_{G}, \mu, H\right)$ is spanned by elements $u \mapsto a(u) l$ with $a \in$ $L^{2}\left(U_{G}, \mu\right)$ and $l \in H$, every element $j \in L^{2}\left(U_{G}, \mu, H\right)$ is a limit a.e. of linear combinations of elements $u \mapsto a(u) l$ [5, Proposition 5, p. 146, and Corollaire, p. 153]. Since $(X(x) a(d(x)) l, b(r(x)) m)=a(d(x)) b(r(x))(X(x) l, m)$, $x \mapsto(X(x) j(d(x)), k(r(x)))$ is Borel for all $j, k \in L^{2}\left(U_{G}, \mu, H\right) .\left|\left(X_{f}, j, k\right)\right| \leqslant$ $\int|f(x)|\|j(d(x))\|\|k(r(x))\| d v(x) \leqslant\|f\|_{I I}\|j\|\|k\|$.

LEMMA 3.2. If $\left(u \mapsto k_{u}\right) \in \int L^{2}\left(G, \lambda^{u}, H\right) d \mu(u), j \in L^{2}\left(U_{G}, \mu, H\right), f \in$ $I I(G)$, and $X$ is as in Lemma 3.1, then

$$
\int\left(\left(X_{f}(j)\right)(u), k_{u}\right)_{u} d \mu(u)=\int f(y)\left(X(y) j(d(y)), k_{u}\right)_{u} d \nu^{u}(y) d \mu(u) .
$$

Proof. Because of the isomorphism $\int L^{2}\left(G, \lambda^{u}, H\right) d \mu(u) \cong$ $L^{2}\left(G, \int \lambda^{u} d \mu(u), H\right)$, there is $k \in L^{2}\left(G, \int \lambda^{u} d \mu(u), H\right)$ such that $\int \| k-$ $k_{u} \|_{u}^{2} d \mu(u)=0$. Hence we may work with $k$. Suppose $k=a l$ with $a \in$ $L^{2}\left(G, \int \lambda^{u} d \mu(u)\right), l \in H$. Then

$$
\begin{aligned}
\iint f(y)(X(y) & j(d(y)), k)_{u} d \nu^{u}(y) d \mu(u) \\
= & \iint f(y) \int(X(y) j(d(y)), a(x) l) d \lambda^{u}(x) d \nu^{u}(y) d \mu(u) \\
& =\iint f(y)(X(y) j(d(y)), l) \int \overline{a(x)} d \lambda^{u}(x) d \nu^{u}(y) d \mu(u) \\
& =\left(X_{f}(j), u \mapsto\left(\int a(x) d \lambda^{u}(x) l\right)\right)_{L^{2}\left(U_{\sigma}, \mu, H\right)} \\
& =\int\left(X_{f}(j)(u),\left(\int a(x) d \lambda^{u}(x)\right) l\right) d \mu(u) \\
& =\iint\left(X_{f}(j)(u), a(x) l\right) d \lambda^{u}(x) d \mu(u) \\
& =\int\left(X_{f}(j)(u), k\right)_{u} d \mu(u) .
\end{aligned}
$$

Thus, the desired result is true for $k$ of the form $u \mapsto a(u) l$; and by linearity 
the result holds for linear combinations. Since the linear span of elements $u \mapsto a(u) l$ is dense in $L^{2}\left(G, \int \lambda^{u} d \mu(u), H\right)$ and the functionals $k \mapsto$ $\int\left(X_{f}(j)(u), k\right)_{u} d \mu(u)$ and $k \mapsto \int f(y)(X(y) j(d(y)), k) u d \nu^{u}(y) d \mu(u)$ are continuous (as in Lemma 3.1), the equality is valid for all $k \in$ $L^{2}\left(G, \int \lambda^{u} d \mu(u), H\right)$.

LEMMA 3.3. Let $X$ as in Lemma 3.1 satisfy $X\left(y^{-1}\right)=X(y)^{*} \nu$-a.e. Suppose $f \in I I(G)$. Then $X_{f^{*}}=\left(X_{f}\right)^{*}$. If $g \in L^{\infty}(G, \nu)$ and vanishes a.e. off a set $E(a), a \geqslant 1$ (see Lemma 2.6), then

$$
\left(X_{f} X_{g}(j), k\right)=\int f(y) \int g(x)(X(y) X(x) j(d(x)), k(r(y))) d \nu^{d(y)}(x) d \nu(y)
$$

and

$$
\left(X_{f * g}(j), k\right)=\int f(y) \int g(x)(X(y x) j(d(x)), k(r(y))) d \nu^{d(y)}(x) d \nu(y) .
$$

Proof.

$$
\begin{aligned}
\left(X_{f^{*}}(j), k\right) & =\int \overline{f\left(y^{-1}\right)} \Delta(y)^{-1}(X(y) j(d(y)), k(r(y))) d \nu(y) \\
& =\int \overline{f(y)}\left(X\left(y^{-1}\right) j(r(y)), k(d(y))\right) d \nu(y) \\
& =\int f(y)\left(X\left(y^{-1}\right)^{*} k(d(y)), j(r(y))\right) d \nu(y)- \\
& =\left(X_{f}(k), j\right)=\left(\left(X_{f}\right)^{*}(j), k\right) .
\end{aligned}
$$

By Lemma 2.6, $g \in I(G)$. On $L^{2}\left(G, \int \lambda^{u} d \mu(u)\right.$; $\left.H\right)$ define an operator $D$ by $\left(D j_{1}, k_{1}\right)=\iint g(x) P(x)\left(X(x) j_{1}(x), k_{1}(x)\right) d \lambda^{u}(x) d \mu(u)$. D commutes with multiplication by elements of $L^{\infty}(G, \nu)$, so that in particular $D=$ $\int D(u) d \mu(u)$ is decomposable as an operator on the direct integral Hilbert space $\int L^{2}\left(G, \lambda^{u}, H\right) d \mu(u) \cong L^{2}\left(G, \int \lambda^{u} d \mu(u), H\right)$. Clearly

$$
\begin{aligned}
\left(D(u) j_{1}, k_{1}\right)_{u}=\int g(x) P(x)\left(X(x) j_{1}(x), k_{1}(x)\right) d \lambda^{u}(x) \quad \text { a.e. } \\
\left(X_{f} X_{g} j, k\right)=\left(X_{g}(j), X_{f^{*}}(k)\right) \\
=\iint g(x) P(x)\left(X(x) j(d(x)), X_{f^{*}}(k)(r(x))\right)_{u} d \lambda^{u}(x) d \mu(u) \\
=\left(D(j \circ d),\left(X_{f^{*}}(k)\right) \circ r\right) \\
=\left(\int\left(X_{f^{*}}(k)(u), D(u)(j \circ d)\right)_{u} d \mu(u)\right) \\
=\left(\iint f^{*}(y)(X(y) k(d(y)), D(u)(j \circ d))_{u} d \nu^{u}(y) d \mu(u)\right)
\end{aligned}
$$


by Lemma 3.2. Thus

$$
\begin{aligned}
\left(X_{f} X_{g}(j), k\right) & =\int f\left(y^{-1}\right) \Delta(y)^{-1} \int g(\dot{x}) P(x) \\
\cdot(X(x) j(d(x)), X(y) k(d(y))) d \lambda^{u}(x) d \nu(y) & \\
= & \int f(y) \int g(x)(X(y) X(x) j(d(x)), k(r(y))) d \nu^{d(y)}(x) d \nu(y) . \\
\left(X_{f * g}(j), k\right) & =\int f * g(x)(X(x) j(d(x)), k(r(x))) d \nu(x) \\
= & \iint f(x y) g\left(y^{-1}\right)(X(x) j(d(x)), k(r(x))) d \nu^{d(x)}(y) d \nu(x) \\
= & \iint f(y) \int g\left(y^{-1} x\right)(X(x) j(d(x)), k(r(x))) d \nu^{u}(x) d \nu^{u}(y) d \mu(u) \\
= & \int f(y) \int g(x)(X(y x) j(d(x)), k(r(y))) d \nu^{d(y)}(x) d \nu(y) .
\end{aligned}
$$

We define now the natural representation $M_{r}$ of $L^{\infty}\left(U_{G}, \mu\right)$ on $L^{2}\left(U_{G}, \mu, H\right)$ by multiplication operators. Explicitly, if $\alpha \in L^{\infty}\left(U_{G}, \mu\right)$ and $j \in L^{2}\left(U_{G}, \mu, H\right)$, then $M_{r}(\alpha) j=(\alpha \circ r) j . M_{r}$ corresponds to the projectionvalued measure used in the inducing construction in group representations.

THEOREM 3.4. If $X$ is a representation of $(G, C)$ on the separable Hilbert space $H$, then $f \mapsto X_{f}$ is a nondegenerate norm-decreasing *-representation of $I I(G)$ on $L^{2}\left(U_{G}, \mu, H\right)$ possessing the following two properties:

(1) For $l, m \in H,\left|\left(X_{f}(u \mapsto l),(u \mapsto m)\right)\right| \leqslant\|f\|_{1}\|l\|\|m\|$,

(2) $M_{r}(\alpha) X_{f}=X_{f(\alpha \circ r)}$.

REMARK 3.5. $f \mapsto X_{f}$ is called the representation of $I I(G)$ induced by the representation $X$ of $G$ (cf. Remark 3.6). The theorem has a converse: any nondegenerate *-representation of $I I(G)$ on $L^{2}\left(U_{G}, \mu, H\right)$ having the two properties in the theorem is induced. Moreover, the same holds for any suitably large separable *-subalgebra of $I(G)$ (for the theorem to make sense, $\alpha$ in property (2) must be restricted appropriately). Here, "suitably large" means that the subalgebra contains the characteristic functions of all elements in a countable generating algebra for $\mathscr{B}(E(u)), u=1,2,3, \ldots$, and that it is closed under $f \mapsto f 1_{F} \circ r$ for $F$ in a countable generating algebra for $\mathscr{B}\left(U_{G}\right)$. Such subalgebras always exist. We will not prove the converse assertions here, because in studying the regular representation one can make explicit constructions to show that certain representations are induced. Minor modification of the proof of Theorem 5.4 of [11] gives the general result. In Example 7.9 of [11] we have given an example of a suitably large subalgebra with a nondegenerate *-representation unitarily equivalent to no induced representation. We will show in the proof of Theorem 5.4 that 
the restriction of the representation $f \rightarrow X_{f}$ to any suitably large subalgebra is also nondegenerate.

Proof (of Theorem 3.4). By Lemma 3.1, $\left\|X_{f}\right\| \leqslant\|f\|_{I I}$. By Lemma 3.3, $\left(X_{f}\right)^{*}=\left(X_{f^{*}}\right)$. If $g \in L^{\infty}(G, v)$ and $g$ vanishes off $E(a)$, then $\left(X_{f * g}(j), k\right)=$ $\left(X_{f} X_{g}(j), k\right)$ by Lemma 3.3. For general $g \in I I(G)$, find $g_{n} \in L^{\infty}(G, \nu)$ with $0<\left|g-g_{n}\right| \leqslant g, g_{n}$ vanishing off $E(n)$, and $\left|g-g_{n}\right| \rightarrow 0$ pointwise. By the Dominated Convergence Theorem, $\left(X_{f *\left(g-g_{n}\right)}(j), k\right)$ and $\left(X_{f} X_{\left(g-g_{n}\right)}(j), k\right)$ converge to zero, so $X_{f * g}=X_{f} X_{g}$.

Let $j$ be a nonzero element of $L^{2}\left(U_{G}, \mu, H\right)$ and let $l_{1}, l_{2}, \ldots$ be an orthonormal basis for $H$. Then $x \mapsto\left(X(x) j(d(x)), l_{i}\right)$ is Borel and for $x$ s.t. $j(d(x)) \neq 0$ there exists $i$ such that $\left(X(x) j(d(x)), l_{i}\right) \neq 0$. It follows that there is an index $i$ and a nonnull Borel set $F \subset G$ such that on $F x \mapsto$ $\left(X(x) j(d(x)), l_{i}\right)$ has real part or imaginary part which is strictly of one sign. Let $f_{n}=1_{F} 1_{E(n)}$. For some $n,\left(X_{f_{n}}(j), \quad\left(u \mapsto l_{i}\right)\right)=$ $\int f_{n}(x)\left(X(x) j(d(x)), l_{i}\right) d \nu(x) \neq 0$. By the Caratheodory Extension Theorem, there is in any generating algebra for $E(n)$ a set $E$ such that $\left(X_{1_{E}} j, l_{i}\right) \neq 0$.

To verify properties (1) and (2), note that

$$
\begin{aligned}
\mid\left(X_{f}(u\right. & \mapsto l),(u \mapsto m)) \mid \\
& =\left|\int f(x)(X(x) l, m) d \nu(x)\right| \leqslant \int|f(x)|\|l\|\|m\| d \nu(x)
\end{aligned}
$$

and

$$
\begin{aligned}
\left(M_{r}(\alpha) X_{f}(j), k\right) & =\left(X_{f}(j), M_{r}(\bar{\alpha})(k)\right) \\
& =\int f(x)(X(x) j(d(x)), \bar{\alpha}(r(x)) k(r(x))) d \nu(x) \\
& =\int f(x) \alpha(r(x))(X(x) j(d(x)), k(r(x))) d \nu(x) .
\end{aligned}
$$

REMARK 3.6. In the situation described in Example 1.11, any representation $f \mapsto T(f)$ of $I I(S \times G)$ induces a representation $g \mapsto T(\Phi(g))$ of $L^{1}(G)$. If $X$ is a representation of $S \times G$, then the inducing construction of Mackey yields a representation $U$ of $G$ on $L^{2}\left(U_{G}, \mu, H\right)$ given by $\left(U_{x} j\right)(s)=$ $\Delta(s, x)^{-1 / 2} \delta(x)^{1 / 2} X(s, x) j(s x)$. The corresponding representation $g \mapsto U_{g}$ of $L^{1}(G)$ is given by

$$
\begin{aligned}
\left(U_{g}(j), k\right) & =\int g(x)\left(U_{x} j, k\right) d h(x) \\
& =\int g(x) \Delta(s, x)^{-1 / 2} \delta(x)^{1 / 2}(X(s, x) j(s x), k(s)) d \mu \times h(s, x) \\
& =\int \Phi g(s, x)(X(s, x) j(s x), k(s)) d \mu \times h(s, x) \\
& =\left(X_{\Phi(g)}(j), k\right) .
\end{aligned}
$$


Thus, there is a commutative diagram

$$
\begin{array}{ccc}
\operatorname{Rep}(I I(S \times G)) & \rightarrow \mapsto T \circ \Phi & \operatorname{Rep}\left(L^{1}(G)\right) \\
\text { inducing } \uparrow & & \uparrow \text { classica } \\
\operatorname{Rep}(S \times G) & \text { inducing } & \operatorname{Rep}(G)
\end{array}
$$

which justifies use of the term "inducing" for the construction described in Theorem 3.4. The left arrow, rather than the top arrow, receives the name because inducing entails manufacturing a new family of operators on a new Hilbert space from another on another space. The step $T \rightarrow T \circ \Phi$ merely selects those operators to be associated with the group. In Chapter V of [11] we have used the converse of the present Theorem 3.4 to prove Mackey's Imprimitivity Theorem [16].

Enlargement of our study to $\sigma$-representations, where $\sigma$ is a Borel 2-cocycle of $G$ (see [20, §10]), is included in succeeding sections of this paper. For economy we will include $\sigma$-representations in the following discussion.

Suppose $X$ and $Y$ are $\sigma$-representations of $(G, C)$ on Hilbert spaces $H$ and $K$, respectively. Let $\mu_{0} \in \tilde{C}$ be a fixed probability. A decomposable operator $T=\int T(u) d \mu_{0}(u)$ from $L^{2}\left(U_{G}, \mu_{0}, H\right)$ to $L^{2}\left(U_{G}, \mu_{0}, K\right) \mu_{0}$-intertwines $X$ with $Y$ if $T(r(x)) X(x)=Y(x) T(d(x)) C$-a.e. The $\mu_{0}$-commuting algebra of $X$ is the algebra of operators $\mu_{0}$-intertwining $X$ with $X . X$ is $\mu_{0}$-primary if its $\mu_{0}$-commuting algebra is a factor and $\mu_{0}$-irreducible if its $\mu_{0}$-commuting algebra is one-dimensional. $X$ and $Y$ are $\mu_{0}$-equivalent if a unitary operator $\mu_{0}$-intertwines them. If $\sigma \equiv 1$, these definitions are for ordinary representations of $G$. See [19].

LEMMA 3.7. Equivalence of $\sigma$-representations $X$ and $Y$ does not depend upon choice of $\mu_{0} \in \tilde{C} . j \mapsto\left(\left(\sqrt{d \mu_{0} / d \mu_{1}}\right) \circ r\right) j$ defines a spacial isomorphism of the $\mu_{0}$ - and $\mu_{1}$-commuting algebras of $X$. If $X$ and $Y$ are equivalent, then their commuting algebras are equivalent via a decomposable unitary operator.

We omit the easy proof. In view of the lemma, we will refer to the commuting algebra, equivalence, primary, and irreducible without mentioning $\mu_{0}$.

THEOREM 3.8. Let $X$ and $Y$ be ordinary representations of $G$. The strong closure of $\left\{X_{f}: f \in I I(G)\right\}$ contains the multiplication operators $\left\{M_{r}(\alpha)\right.$ : $\left.\alpha \in L^{\infty}\left(U_{G}, \mu\right)\right\}$. An operator $T$ intertwines $X$ with $Y$ iff $T X_{f}=Y_{f} T$ for all $f \in I I(f)$ iff $T X_{f}=Y_{f} T$ for all $f$ in a suitably large *-subalgebra $\mathbb{Q}$ of $I(G)$. The commuting algebra of $X$ is $\left\{X_{f}: f \in \mathbb{Q}\right\}^{\prime}=\left\{X_{f}: f \in I I(G)\right\}^{\prime} . X$ and $Y$ are equivalent iff $f \mapsto X_{f}$ and $f \mapsto Y_{f}$ are unitarily equivalent.

Proof. By Theorem 3.4, $\left\{X_{f}: f \in I I(G)\right\}$ is a nondegenerate *-algebra of 
bounded operators. By Corollaire 2, p. 44 of [5], the identity operator $I$ of $L^{2}\left(U_{G}, \mu, H\right)$ belongs to the strong closure of this algebra. Let $f_{\gamma}$ be a net in $I(G)$ such that $X_{f_{r}} \rightarrow I$ strongly. Then $M_{r}(\alpha)=M_{r}(\alpha) I=\lim M_{r}(\alpha) X_{f_{r}}=$ $\lim \left(X_{f_{r}, \circ}\right) \in\left\{X_{f}: f \in I I(G)\right\}^{\prime \prime}$. Suppose $T X_{f}=Y_{f} T$ for all $f \in \mathbb{Q}$. Then for $j \in L^{2}\left(U_{G}, \mu, H\right)$ and $k \in L^{2}\left(U_{G}, \mu, K\right), f \mapsto\left(\left(T X_{f}-Y_{f} T\right) j, k\right)$ extends to be the zero integral, because $\mathscr{Q}$ contains characteristic functions of generating algebras for $\mathscr{B}(E(n)), n=1,2, \ldots$ Thus $T X_{f}=Y_{f} T$ for all $f \in I I(G)$. Hence $\lim \left(Y_{f_{r}} T\right)=\lim \left(T X_{f_{r}}\right)=T$ and $T M_{r}(\alpha)=\lim \left(T X_{f_{r} \alpha, r}\right)=$ $\lim \left(Y_{f_{\gamma} \alpha, r} T\right)=\lim \left(M_{r}(\alpha) Y_{f_{r}} T\right)=M_{r}(\alpha) T$, so $T$ is decomposable, $T=$ $\int T(u) d \mu(u)$. Since

$$
\begin{aligned}
& \int 1_{E}(x)(T(r(x)) X(x) j(d(x)), k(r(x))) d \nu(x) \\
&=\left(X_{1_{E}} j, T^{*} k\right)=\left(Y_{1_{E}} T(j), k\right) \\
&= \int 1_{E}(x)(Y(x) T(d(x)) j(d(x)), k(r(x))) d \nu(x)
\end{aligned}
$$

for all $E$ belonging to a countable generating algebra of $\mathscr{B}(E(n)), n=$ $1,2, \ldots, T(r(x)) X(x)=Y(x) T(d(x)) \nu$-a.e. The remaining assertions are easily verified.

It is a consequence of direct integral theory that the function $u \mapsto$ $\operatorname{dim} L^{2}\left(G, \nu^{u}\right)$ is measurable on $U_{G}$. A different choice of Haar measure changes this function only on a null set.

Definition 3.9. $(G, C)$ is homogeneous if $\operatorname{dim} L^{2}\left(G, \nu^{u}\right)$ is essentially constant.

LEMMA 3.10. There is a sequence $G_{\infty}, G_{1}, G_{2}, \ldots$ of disjoint reductions of $G$ such that $\operatorname{dim} L^{2}\left(G, \nu^{u}\right)=\operatorname{dim} L^{2}\left(G_{i}, \nu^{u}\right)=i$ a.e. on $U_{G} \cap G_{i}$ and $\cup G_{i}=G$ a.e. The sequence $G_{\infty}, G_{1}, G_{2}, \ldots$ is determined uniquely up to null sets. If $\mu\left(U_{G} \cap G_{i}\right)=c_{i}>0$, then $\left(\nu\left|G_{i}, c_{i}^{-1} \mu\right|\left(U_{G} \cap G_{i}\right)\right.$ is a Haar measure for $G_{i}$ and $\Delta \mid G_{i}$ is its modular function.

Proof. Let $F(i) \subset U_{G}, i=\infty, 1,2, \ldots$, be Borel sets such that $u \in F(i)$ $\Rightarrow \operatorname{dim} L^{2}\left(G, \nu^{u}\right)=i$ and $\operatorname{dim} L^{2}\left(G, \nu^{u}\right) \neq i$ for a.e. $u \in U_{G}-F(i)$. We can find $U_{0} \subset \cup F(i)$ conull Borel such that $\lambda^{u}\left(G \mid U_{0}\right)=1$ if $n \in U_{0}$. Then $x \in G \mid U_{0} \Rightarrow \nu^{r(x)}=\left(E \mapsto \int 1_{E}(x y) d \nu^{d(x)}(y)\right)$ as measures on $G \mid U_{0}$ and $u \mapsto \operatorname{dim} L^{2}\left(G \mid U_{0}, \nu^{u}\right)$ is Borel. Thus we may assume $\operatorname{dim} L^{2}\left(G, \nu^{u}\right)$ is Borel and $F(i)=\left\{u: \operatorname{dim} L^{2}\left(G, \nu^{u}\right)=i\right\}$. Clearly $r(x) \in F(i) \Rightarrow d(x) \in F(i)$; that is, $F(i)$ are saturated. Hence $u \in F(i) \Rightarrow G \mid F(i)$ is $\nu^{u}$-conull and $u \in U_{G}-$ $F(i) \Rightarrow G \mid F(i)$ is $\nu^{u}$-null. $G_{i}=G \mid F(i)$ are the reductions desired. The $r$ decomposition of $\nu \mid G_{i}$ with respect to $c_{i}^{-1} \mu \mid F(i)$ is $\nu \mid G_{i}=\int_{F(i)} c_{i} \nu^{u} c_{i}^{-1} d \mu(u)$, so $\left(\nu\left|G_{i}, \quad c_{i}^{-1} \mu\right| F(i)\right)$ is a Haar measure. $\int f\left(x^{-1}\right) 1_{F(i)}(d(x)) d \nu(x)=$ $\int f(x) \Delta(x)^{-1} 1_{F(i)}(r(x)) d v(x)$, so $\Delta \mid G_{i}$ is the modular homomorphism. 
For ergodic groupoids there can be no nontrivial decomposition as described in the lemma, so every ergodic groupoid is homogeneous. Homogeneous groupoids are exactly the class of groupoids of which we can describe a representation inducing (up to equivalence) the regular representation of $I I(G)$. The construction we give is an adaptation of one made by Ramsay [22].

EXAMPLE 3.11. Assume $(G, C)$ is a homogeneous groupoid and $\sigma$ is a strict 2-cocycle [20, p. 312]. Let $H$ be a fixed Hilbert space to which a.a. $L^{2}\left(G, \nu^{u}\right)$ are isomorphic. There is an isomorphism $V=\int V(u) d \mu(u)$ of $\int L^{2}\left(G, \nu^{u}\right) d \mu(u) \cong L^{2}(G, \nu)$ with $L^{2}\left(U_{G}, \mu, H\right)$. We may assume that $u \mapsto$ $V(u) f$ is Borel if $f$ is Borel. Define $W_{\sigma}(X): L^{2}\left(G, \nu^{d(x)}\right) \rightarrow L^{2}\left(G, \nu^{r(x)}\right)$ by

$$
W_{\sigma}(x)(f)(y)=\sigma\left(y^{-1}, x\right)^{-1} f\left(x^{-1} y\right) .
$$

By the cocycle identity,

$$
\begin{aligned}
W_{\sigma}(x) W_{\sigma}(y) f(z) & =\sigma\left(z^{-1}, x\right)^{-1} \sigma\left(z^{-1} x, y\right)^{-1} f\left(y^{-1} x^{-1} z\right) \\
& =\sigma\left(z^{-1}, x y\right)^{-1} \sigma(x, y)^{-1} f\left(y^{-1} x^{-1} z\right) \\
& =\sigma(x, y)^{-1} W_{\sigma}(x y) f(z) .
\end{aligned}
$$

Let $U_{1} \subset U_{G}$ be a conull Borel set on which $V(u)$ is unitary. Define $W^{\sigma}(x)=V(r(x)) W_{\sigma}(x) V(d(x))^{-1}$ on $G \mid U_{1}$ and $W^{\sigma}(x)=I$ otherwise. $W^{\sigma}$ is a $\sigma$-representation, which we call the $\sigma$-regular representation of $(G, C)$. Up to equivalence, $W^{\sigma}$ does not depend upon the choice of $V . W^{\sigma}$ is strict on the i.r. $G \mid U_{1}$.

In the special case $\sigma \equiv 1, W=W^{1}$ is the regular representation of $G$. Suppose $j, k \in L^{2}(G, v) \cong \int L^{2}\left(G, \nu^{u}\right) d \mu(u)$.

$$
\begin{aligned}
\left(W_{f} V(j), V(k)\right) & =\int f(x)(W(x) V(j)(d(x)), V(k)(r(x))) d \nu(x) \\
& =\int f(x)\left(V^{-1}(r(x)) W(x) V(d(x)) j, k\right)_{r(x)} d \nu(x) \\
& =\iint f(x)\left(W_{1}(x) j, k\right)_{u} d \nu^{u}(x) d \mu(u) \\
& =\iint f(x) \int j\left(x^{-1} y\right) \overline{k(y)} d \nu^{r(x)}(y) d \nu^{u}(x) d \mu(u) \\
& =\left(L_{f}(j), k\right) .
\end{aligned}
$$

Thus $L$ and $f \mapsto W_{f}$ are unitarily equivalent. This fact, together with Lemma 3.7 and the following result, frees the left von Neumann algebra described in $\$ 2$ from seeming dependence on the Haar measure.

Proposition 3.13. Up to equivalence $W^{\sigma}$ is independent of the Haar measure. 
Proof. If $\left(\nu^{0}, \mu^{0}\right)$ is another Haar measure and $\psi^{0}=d \mu^{0} / d \tilde{\lambda}$ and $P^{0} \psi^{0} \circ r$ $=d \nu^{0} / d \lambda$, then by Corollary 3.14 of [12] there is a positive Borel function $\phi$ on $U_{G}$ such that a.e. $P^{0}=P \phi \circ d$. Let $\left(W_{0}\right)^{0}$ be as defined by (3.12) using $\left(\nu^{0}, \mu^{0}\right)$. Let $V^{0}=\int V^{0}(u) d \mu^{0}(u): L^{2}\left(G, \nu^{0}\right) \rightarrow L^{2}\left(U_{G}, \mu^{0}, H\right)$ be defined by $V^{0}(u) f=V(u)\left(f \sqrt{\phi^{\circ} d}\right)$. Use $V^{0}$ to form $\left(W^{0}\right)^{0}$; we have remarked already that up to equivalence $\left(W^{\sigma}\right)^{0}$ is independent of the choice of $V^{0}$. Let $f, g \in L^{2}(G, \nu)$.

$$
\begin{aligned}
\left(\left(W^{\sigma}\right)^{0}(x) V^{0}(d(x)) f, V^{0}(r(x)) g\right)_{r(x)} & \\
= & \left(\left(W_{\sigma}\right)^{0}(x) f, g\right)=\int \sigma\left(y^{-1}, x\right)^{-1} f\left(x^{-1} y\right) \overline{g(y)} d\left(\nu^{0}\right)^{r(x)}(y) \\
& =\int \sigma\left(y^{-1}, x\right)^{-1} f\left(x^{-1} y\right) \sqrt{\phi^{\circ} d\left(x^{-1} y\right)} \overline{g(y) \sqrt{\phi^{\circ} d(y)}} d \nu^{r(x)}(y) \\
& =\left(W_{o}(x)\left(f \sqrt{\phi^{\circ} d}\right), g \sqrt{\phi^{\circ} d}\right)_{r(x)} \\
& =\left(W^{o}(x) V^{0}(d(x)) f, V^{0}(r(x)) g\right)_{r(x)}
\end{aligned}
$$

Thus $W^{\sigma}=\left(W^{\sigma}\right)^{0}$ for this choice of $V^{0}$.

4. The twisted regular representation. For a given strict 2-cocycle $\sigma$ of the homogeneous groupoid $(G, C)$, we have constructed in Example 3.11 a $\sigma$-representation $W^{\sigma}$ which is called the $\sigma$-regular representation. By Proposition 3.14, the equivalence class of $W^{\sigma}$ among all $\sigma$-representations is uniquely determined, so by Lemma 3.7 there is a von Neumann algebra $\left(W^{\sigma}\right)^{\prime}$ associated with $(G, C)$ and $\sigma$. When $\sigma \equiv 1$, considerably more has already been demonstrated: $W^{\prime}$ is the commutant of the regular representation of $I I(G)$ and is in standard form, $W^{\prime \prime}$ being generated by $\mho_{I}$; $f \mapsto \Delta^{z} f$ is the modular automorphism group and

$$
J f=\left(x \mapsto \Delta(x)^{-1 / 2} \overline{f\left(x^{-1}\right)}\right)
$$

defines the modular involution. This information is useful in determining, for example, whether $W^{\prime}$ is semifinite (see §5); therefore this section is devoted to extending the analysis of the ordinary regular representation to $\sigma$-regular representations.

Let $(G, C)$ be a measure groupoid, not necessarily homogeneous. The device used for deriving the information needed is the groupoid extension $G^{\sigma}$ [22], [30]. Let $\mathbf{T}$ be the unit circle with normalized Lebesgue measure $m$. The set $G^{\boldsymbol{\sigma}}=G \times \mathbf{T}$ is endowed with groupoid structure by defining $(x, s)(y, t)$ $=\left(x y, \operatorname{st} \sigma(x, y)^{-1}\right)$ for $(x, y) \in G^{(2)}$ and $(x, s)^{-1}=\left(x^{-1}, s^{-1} \sigma\left(x, x^{-1}\right)\right)$. Note that $\sigma\left(x, x^{-1}\right)=\sigma\left(x, x^{-1}\right) \sigma\left(x x^{-1}, x\right)=\sigma\left(x, x^{-1} x\right) \sigma\left(x^{-1}, x\right)=$ $\sigma\left(x^{-1}, x\right) . C^{\sigma}=C \times[m] .\left(\nu \times m, \mu \times \delta_{1}\right)$ is a Haar measure. $(x, s) \mapsto \Delta(x)$ is the modular homomorphism. 
If $X$ is a $\sigma$-representation of $(G, C)$ on $H$, then $\tilde{X}=((x, s) \mapsto s X(x))$ is an ordinary representation of $\left(G^{\sigma}, C^{\sigma}\right)$. In particular, if $G$ is homogeneous, then $\tilde{W}^{\sigma}$ is an ordinary representation of $G^{\sigma}$. For the following proposition, we adopt the notation $X_{f}$ from Lemma 3.1; $\left(U_{G} \times\{1\}, \mu \times \delta_{1}\right)$ is identified with $\left(U_{G}, \mu\right)$.

THEOREM 4.1. The commuting algebras of $X$ and of $\tilde{X}$ coincide. The assertions of Theorem 3.8 are valid for $\sigma$-representations $X$ and $Y$.

Proof. Since $r(x, s)=(r(x), 1)$ and $d(x, s)=(d(x), 1)$, the first statement follows from the definitions. The second statement follows from the proof of Theorem 3.8, provided it is shown that there is a net $f_{\gamma} \in I I(G)$ such that $X_{f_{\gamma}} \rightarrow I$ strongly. By the proof of Theorem 3.8, there is a net $g_{\gamma} \in I I\left(G^{\sigma}\right)$ such that $\tilde{X}_{g_{\gamma}} \rightarrow I$ strongly. Let $f_{\gamma}(x)=\int s g_{\gamma}(x, s) d m(s) . f_{\gamma} \in I I(G)$. For $j$, $k \in L^{2}\left(U_{G}, \mu, H\right)$,

$$
\begin{aligned}
\left(X_{f} j, k\right) & =\int\left(\int s g_{\gamma}(x, s) d m(s)\right)(X(x) j(d(x)), k(r(x))) d \nu(x) \\
& =\iint g_{\gamma}(x, s)(s X(x) j(d(x)), k(r(x))) d \nu \times m(x, s) \\
& =\left(\tilde{X}_{g_{\gamma}} j, k\right) .
\end{aligned}
$$

Therefore, $\lim X_{f_{y}}=\lim \tilde{X}_{g_{y}}=I$.

In what follows the symbol $\sim$ will be used to denote functions on $G^{\sigma}$. For $\tilde{g} \in L^{2}\left(G^{o}, \nu \times m\right)$ define $B \tilde{g}(x, s)=s^{-1} \int t \tilde{g}(x, t) d m(t)$. Computation shows that $B$ is a projection. Let $\tilde{f} \in \mathcal{U}_{I}\left(G^{\sigma}\right)$. For a.a. $(x, s)$,

$$
\begin{aligned}
& \tilde{f} * B \tilde{g}(x, s)=\iint \tilde{f}(y, q) B \tilde{g}\left(y^{-1} x, q^{-1} s \sigma\left(y^{-1}, y\right) \sigma\left(y^{-1}, x\right)^{-1}\right) \\
& \cdot d \nu^{r(x)}(y) d m(q) \\
&= \iint \tilde{f}(y, q) \sigma\left(y^{-1}, x\right) q s^{-1} \sigma\left(y^{-1}, y\right)^{-1} \int t \tilde{g}\left(y^{-1} x, t\right) d m(t) \\
& \cdot d \nu^{r(x)}(y) d m(q) \\
&= s^{-1} \int t \iint \tilde{f}(y, q) \tilde{g}\left(y^{-1} x, q^{-1} t \sigma\left(y^{-1}, y\right) \sigma\left(y^{-1}, x\right)^{-1}\right) \\
& \cdot d \nu^{r(x)}(y) d m(q) d m(t) \\
&= s^{-1} \int t \tilde{f} * \tilde{g}(x, t) d m(t)=B(\tilde{f} * \tilde{g})(x, s) .
\end{aligned}
$$

This shows that $B$ commutes with the regular representation $\tilde{L}$ of $G^{\circ}$. The modular involution associated with $\mathscr{U}_{I}\left(G^{\circ}\right)$ is given by $\tilde{J} \tilde{f}(x, s)=$ $\Delta(x)^{-1 / 2}\left(\tilde{f}\left(x^{-1}, s^{-1} \sigma\left(x^{-1}, x\right)\right)\right)^{-}$. A computation shows that $J B J \tilde{f}=B \tilde{f}$. Thus $B$ is a central projection of $\tilde{L}$. 
Let $K: \quad L^{2}(G, \nu) \rightarrow L^{2}\left(G^{\sigma}, \nu \times m\right)$ be defined by $(K f)(x, s)=$ $s^{-1} \sigma\left(x^{-1}, x\right) f(x) .\|K f\|_{2}=\|f\|_{2}$. We claim $B$ is projection on the image of $K$. Clearly $B K f=K f$. If $\tilde{f} \in L^{2}\left(G^{\sigma}, \nu \times m\right)$, let $f(x)=$ $\sigma\left(x^{-1}, x\right)^{-1} \int t \tilde{f}(x, t) d m(t)$. Then $K f(x, s)=s^{-1} \int t \tilde{f}(x, t) d m(t)=B \tilde{f}(x, s)$. This proves the claim. $K: L^{2}(G, \nu) \rightarrow B L^{2}\left(G^{\sigma}, \nu \times m\right)$ is an isomorphism.

Let $L_{\tilde{f}}$ be the restriction of $\tilde{L}_{\tilde{f}}$ to the range of $B$. The restriction $J$ of $\tilde{J}$ to the range of $B$ is a conjugate linear involutive isometry. Let $\tilde{L}^{\prime}$ and $L^{\prime}$ be the respective commuting algebras. For $f \in I I(G)$ define $T_{f}$ on $L^{2}(G, \nu)$ by

$$
\begin{gathered}
\left(T_{f} j, k\right)=\iint f(x) \sigma\left(y^{-1}, x\right)^{-1} j\left(x^{-1} y\right) \overline{k(y)} d \nu^{r(x)}(y) d \nu(x) \\
\text { for } j, k \in L^{2}(G, \nu) .
\end{gathered}
$$

The proof of the next theorem shows that $T_{f}$ is a bounded operator.

THEOREM 4.3. $B \bigcup_{I}\left(G^{\sigma}\right)$ is a modular Hilbert algebra whose left von Neumann algebra is $L^{\prime \prime}$, modular involution $J$, and commutant $L^{\prime}=J L^{\prime \prime} J . L^{\prime \prime}$ is spacially isomorphic to the weak closure of $\left\{T_{f}: f \in I I(G)\right\}$. Up to decomposable spacial isomorphism, $L^{\prime \prime}$ and $J$ do not depend upon the Haar measure $(\nu, \mu)$. If $G$ is homogeneous, with $\sigma$-regular representation $W^{\sigma}$, then $L$ is unitarily equivalent to $\tilde{f} \mapsto \tilde{W}_{\tilde{f}}^{\tilde{s}}$ and $L^{\prime \prime}$ is spacially isomorphic over $M_{r}\left(L^{\infty}\left(U_{G}, \mu\right)\right)$ to the commutant of the commuting algebra of $W^{\sigma}$.

Proof. If $\tilde{f} \in L^{2}\left(G^{\sigma}, \nu \times m\right) \cap I\left(G^{\sigma}\right)$ and is $\Delta$-bounded, then so is $B \tilde{f}$.

$$
\begin{aligned}
(B \tilde{f})^{*}(x, s) & =\Delta(x)^{-1} \overline{B \tilde{f}}\left(x^{-1}, s^{-1} \sigma\left(x^{-1}, x\right)\right) \\
& =s^{-1} \sigma\left(x^{-1}, x\right) \Delta(x)^{-1} \int t^{-1} \overline{\tilde{f}\left(x^{-1}, t\right)} d m(t) \\
& =s^{-1} \int t^{-1} \overline{\tilde{f}}\left(x^{-1}, t \sigma\left(x, x^{-1}\right)\right) \Delta(x)^{-1} d m(t) \\
& =B\left(\tilde{f}^{*}\right)(x, s) .
\end{aligned}
$$

Also $B\left(\Delta^{z} \tilde{f}\right)=\Delta^{z} B(\tilde{f})$ and $\left(\left(1+\Delta^{c}\right) B \tilde{f}, B \tilde{g}\right)=\left(\left(1+\Delta^{c}\right) \tilde{f}, B \tilde{g}\right)$.

$$
\begin{aligned}
& ((B \tilde{f}) *(B \tilde{g}), B \tilde{h})=\left(\Delta^{1}(B \tilde{h})^{*},((B \tilde{f}) * B \tilde{g})^{*}\right) \\
& =\left(B\left(\Delta^{1} \tilde{h}^{*}\right), B\left(\tilde{g}^{*}\right) * B\left(\tilde{f}^{*}\right)\right)=\left(B\left(\Delta^{1} \tilde{h}^{*}\right), \tilde{L}_{B\left(\tilde{g}^{*}\right)}\left(B\left(\tilde{f}^{*}\right)\right)\right) \\
& =\left(B\left(\Delta^{1} \tilde{h}^{*}\right), B \tilde{L}_{B\left(\tilde{g}^{*}\right)}\left(\tilde{f}^{*}\right)\right)=\left(B\left(\Delta^{1} \tilde{h}^{*}\right),(\tilde{f} * B \tilde{g})^{*}\right) \\
& =(\tilde{f} * B \tilde{g}, B \tilde{h})=(\tilde{f} * \tilde{g}, B \tilde{h}) .
\end{aligned}
$$

Since $\mathcal{U}_{I}\left(G^{\sigma}\right)$ is a modular Hilbert algebra, these facts imply that $B \bigcup_{I}\left(G^{\sigma}\right)$ is a modular Hilbert algebra. It is clear from the definitions and the fact that $\tilde{J}$ commutes with $B$ that $J$ is the modular involution associated with $B$ थ $_{I}\left(G^{\sigma}\right)$. 
Since $((B \tilde{f}) *(B \tilde{g}), B \tilde{h})=\left(\tilde{L}_{B \tilde{f}}(B \tilde{g}), B \tilde{h}\right), L^{\prime \prime}$ contains the left von Neumann algebra of $B \mathscr{Q}_{I}(G)$. By Theorem $2.15, \tilde{L}^{\prime \prime}$ is generated by $\left\{\tilde{L_{\tilde{f}}}: \tilde{f} \in \mathcal{U}_{I}\left(G^{\sigma}\right)\right\}$. Hence $L^{\prime \prime}$ is generated by $\left\{L_{\tilde{f}} \tilde{f} \in \mathcal{f}_{I}\left(G^{\sigma}\right)\right\}$. Since $L_{\tilde{f}}=L_{B \tilde{f}}$ by (4.4), $L^{\prime \prime}$ is generated by $\left\{L_{\tilde{f}}: \tilde{f} \in B \mathscr{Q}_{I}\left(G^{\sigma}\right)\right\}$.

It follows from the cocycle properties that $\sigma\left(x^{-1}, x\right)^{-1} \sigma\left(x^{-1}, y\right)$ $\cdot \sigma\left(y^{-1} x, x^{-1} y\right) \sigma\left(y^{-1}, y\right)^{-1}=\sigma\left(y^{-1}, x\right)^{-1}$ for $(x, y) \in G^{(2)}$. Using this identity, we calculate that

$$
\begin{gathered}
\left(L_{\tilde{f}} K g, K h\right)=\int \tilde{f}(x, s) \int K g\left(x^{-1} y, s^{-1} t \sigma\left(x, x^{-1}\right) \sigma\left(x^{-1}, y\right)^{-1}\right) \\
\cdot \overline{K h}(y, t) d \nu^{r(x)} \times m(y, t) d \nu \times m(x, s) \\
=\int \tilde{f}(x, s) \int s \sigma\left(x^{-1}, x\right)^{-1} \sigma\left(x^{-1}, y\right) \sigma\left(y^{-1} x, x^{-1} y\right) \\
\cdot \sigma\left(y^{-1}, y\right)^{-1} g\left(x^{-1} y\right) \overline{h(y)} d \nu^{r(x)}(y) d \nu \times m(x, s) \\
=\int \tilde{f}(x, s) \int s \sigma\left(y^{-1}, x\right)^{-1} g\left(x^{-1} y\right) \overline{h(y)} d \nu^{r(x)}(y) d \nu \times m(x, s) .
\end{gathered}
$$

Let $f(x)=\int s \tilde{f}(x, s) d m(s)$. If $\tilde{f} \in I I\left(G^{o}\right)$, then $f \in I I(G)$ and (4.5) shows that $\left(L_{\tilde{f}} K g, K h\right)=\left(T_{f} g, h\right)$. If $f$ is an arbitrary element of $I I(G)$ then $\tilde{f}(x, s)$ $=s^{-1} f(x)$ defines an element of $I I\left(G^{\sigma}\right)$ such that $f(x)=\int s \tilde{f}(x, s) d m(s)$. It follows that $\left\{T_{f}: f \in I I(G)\right\}$ is a ${ }^{*}$-algebra of operators. Since $\left\{L_{\tilde{f}}: \tilde{f} \in\right.$ $\left.I I\left(G^{\circ}\right)\right\}$ is weakly dense in $L^{\prime \prime}$, the weak closure of $\left\{T_{f}: f \in I I(G)\right\}$ and $L^{\prime \prime}$ are isomorphic via the isomorphism $K$ of $L^{2}(G, \nu)$ onto $B L^{2}\left(G^{o}, \nu \times m\right)$. The same holds for $I(G)$ instead of $I I(G)$.

Suppose $G$ is homogeneous with $W^{\sigma}$ the $\sigma$-regular representation. Let $V=\int V(u) d \mu(u)$ as in Example 3.11, so $W^{o}(x)=V(r(x)) W_{o}(x) V(d(x))^{-1}$. We calculate

$$
\begin{aligned}
\left(\tilde{W}_{\tilde{f}}^{o} V g, V h\right) & =\int \tilde{f}(x, s)\left(\tilde{W}^{o}(x, s) V(d(x)) g, V(r(x)) h\right)_{r(x)} d \nu \times m(x, s) \\
& =\int \tilde{f}(x, s)\left(s W_{\sigma}(x) g, h\right)_{r(x)} d \nu \times m(x, s) \\
& =\int \tilde{f}(x, s) s \int \sigma\left(y^{-1}, x\right)^{-1} g\left(x^{-1} y\right) \overline{h(y)} d \nu^{r(x)}(y) d \nu \times m(x, s) \\
& =\left(L_{\tilde{f}} K g, K h\right)
\end{aligned}
$$

by (4.5). Thus $L$ and $\tilde{f} \mapsto \tilde{W}_{\tilde{f}}^{o}$ are unitarily equivalent via a decomposable isometry. From this and Theorem 4.1 it follows that $L^{\prime}$ and $\tilde{W}^{\sigma \prime}$ are spacially isomorphic via an isometry decomposable with respect to $M_{r}\left(L^{\infty}\left(U_{G}, \mu\right)\right)$, so by Theorem 4.1 again the same is true of $L^{\prime}$ and $W^{\circ \prime}$, hence also of $L^{\prime \prime}$ and $W^{o \prime \prime}$. We remark for use later that $K^{-1} J K=J_{\sigma}$ is defined by

$$
J_{o} g(x)=\sigma\left(x^{-1}, x\right)^{-1} \Delta(x)^{-1 / 2} \overline{g\left(x^{-1}\right)} .
$$


All that remains is to prove that $L^{\prime \prime}$ and $J$ are independent of the Haar measure up to a decomposable spacial isometry. Let us continue to assume that $G$ is homogeneous and adopt notation of Proposition 3.13. Let $q(x, s)=$ $\psi \circ r(x)^{1 / 2} \psi^{0} \circ r(x)^{-1 / 2} \phi \circ d(x)^{-1 / 2}$. It follows easily from Lemma 3.7 and the proof of Proposition 3.13 that $j \mapsto q j$ is an isometry of $L^{2}(G \times \mathbf{T}, \nu \times m)$ onto $L^{2}\left(G \times \mathbf{T}, \nu^{0} \times m\right)$ furnishing an isomorphism of $\tilde{L}^{\prime \prime}$ with the regular representation $\left(\tilde{L}^{0}\right)^{\prime \prime}$ constructed from the Haar measure $\left(\nu^{0} \times m, \mu^{0} \times \delta_{1}\right)$. This isometry carries $\tilde{J}$ to its counterpart $\tilde{J}^{0}$ and $B$ to its counterpart $B^{0}$. For $G$ not necessarily homogeneous we appeal to Lemma 3.10 to decompose $G$ as a disjoint union of homogeneous groupoids. $L^{\prime \prime}$ decomposes as a direct sum and we can apply the analysis above to each summand.

REMARK 4.7. For essentially transitive $G$, the ordinary regular representation has been defined by Westman [28, p. 628]. As described in [20, Theorem 6.19], such $G$ is similar to a separable locally compact group $g$ which is determined by $G$ up to isomorphism. It can be shown that the commuting algebra of the regular representation of $G$ is the tensor product of the commuting algebra of the regular representation of $g$ by a factor of type $I$. A proof may be based on Theorem 5.19 of [11] and Theorem 4.4 of [12].

EXAMPLE 4.8. The von Neumann algebra described in Theorem 4.3 is our most extensive generalization of the group-measure space construction. The purpose of this example is to exhibit the relationship between $L^{\prime \prime}$ of Theorem 4.3 and a construction made by Zeller-Meier [31]. References to related constructions may be found in [24] and at the end of $\$ 5$.

Let $g$ be a countable group acting on the analytic probability space $(S, \mu)$. Let $h$ be the counting measure (Haar measure) on g. Let $\delta_{x}^{x_{0}}=1$ if $x=x_{0}$, $\delta_{x}^{x_{0}}=0$ if $x \neq x_{0}$. Let $\alpha \in L^{\infty}(S, \mu)$. Let $\Delta$ be the modular homomorphism for the Haar measure $(\mu \times h, \mu)$. The function $f$ defined by $f(s, x)=$ $\alpha(s) \Delta(s, x)^{-1 / 2} \delta_{x}^{x_{0}}$ belongs to $I I(S \times G, \mu \times h, \mu)$. If $\tau$ is a 2-cocycle for $g$ such that $\tau(e, e)=1$,

$$
((s, x),(s x, y)) \mapsto \tau(x, y) \tau\left(x, x^{-1}\right) \tau\left(y, y^{-1}\right) \tau\left(x y, y^{-1} x^{-1}\right)^{-1}=\sigma(x, y)
$$

defines a strict 2-cocycle $\sigma$ of $S \times \mathrm{g} . S \times \mathrm{g}$ is homogeneous, so we may construct $W_{\sigma}$ as in (3.12) and the $\sigma$-regular representation $W^{\sigma}$, whether $\mu$ is g-ergodic or not.

$$
\begin{aligned}
& \left(T_{f}\left(g_{1}\right), g_{2}\right) \\
& \quad=\int f(s, x) \int \sigma\left(y^{-1}, x\right)^{-1} g_{1}\left(s x, x^{-1} y\right) \overline{g_{2}(s, y)} d h(y) d \mu \times h(s, x) \\
& \quad=\int \sigma\left(y^{-1}, x_{0}\right)^{-1} \alpha(s) \Delta\left(s, x_{0}\right)^{-1 / 2} g_{1}\left(s x_{0}, x_{0}^{-1} y\right) \overline{g_{2}(s, y)} d \mu \times h(s, y) .
\end{aligned}
$$

Setting $x_{0}=e \in g$, we find that $T_{f}=M_{r}(\alpha)$, multiplication by $s \mapsto \alpha(s)$. 
Thus the commutant of $\mathcal{Q}=\left\{T_{f}: f(s, x)=\alpha(s) \Delta(s, x)^{-1 / 2} \delta_{x}^{x_{0}}\right.$ for some $\alpha \in$ $\left.L^{\infty}(S, \mu), x_{0} \in \mathfrak{g}\right\}$ consists of operators decomposable with respect to $M_{r}\left(L^{\infty}(S, \mu)\right)$. If $T=\int T(s) d \mu(s)$ belongs to the commutant of $\mathbb{Q}$, then

$$
0=\int f(s, x)\left(\left(T(s) W_{\sigma}(s, x)-W_{\sigma}(s, x) T(s x)\right) g_{1}, g_{2}\right)_{s} d \mu \times h(s, x)
$$

for all $f$ of the form $\alpha(s) \Delta(s, x)^{-1 / 2} \delta_{x}^{x_{0}}$. Hence for each $x_{0} \in \mathfrak{g}$

$$
\alpha \mapsto \int \alpha(s)\left(\left(T(s) W_{\sigma}\left(s, x_{0}\right)-W_{\sigma}\left(s, x_{0}\right) T\left(s x_{0}\right)\right) g_{1}, g_{2}\right)_{s} d \mu(s)
$$

is the zero integral. Thus $T \in\left\{T_{f}: f \in I I(S \times g)\right\}^{\prime}$, so $\left\{T_{f}: f \in I I(S \times\right.$ g) $\}^{\prime \prime}$ is generated by $\left\{T_{f}: f(s, x)=\alpha(s) \Delta(s, x)^{-1 / 2} \delta_{x}^{x_{0}}\right.$ for $\alpha \in L^{\infty}(S, \mu)$ and $\left.x_{0} \in \mathfrak{g}\right\}$.

Let $J_{\sigma}=K^{-1} J K$ as in (4.6). $J_{\sigma} g(s, x)=\sigma\left(x^{-1}, x\right)^{-1} \Delta(s, x)^{-1 / 2} \overline{g\left(s x, x^{-1}\right)}$. For $f(s, x)=\alpha(s) \Delta(s, x)^{-1 / 2} \sigma\left(x^{-1}, x\right) \delta_{x}^{x_{0}}$ we calculate

$$
\begin{aligned}
J_{\sigma} & T_{f} J_{\sigma} g(s, x) \\
& =\sigma\left(x^{-1}, x\right)^{-1} \Delta(s, x)^{-1 / 2}\left(\int f(s x, y) \sigma(x, y)^{-1} J_{\sigma} g\left(s x y, y^{-1} x^{-1}\right) d h(y)\right) \\
& =\sigma\left(x^{-1}, x\right)^{-1} \Delta(s, x)^{-1 / 2} \int \Delta\left(s x y, y^{-1} x^{-1}\right)^{-1 / 2} \overline{f(s x, y)} \\
& \cdot \sigma(x, y) \sigma\left(x y, y^{-1} x^{-1}\right) g(s, x y) d h(y) \\
& =\int \delta_{y}^{x_{0}}(x, y) \overline{\alpha(s x)} g(s, x y) d h(y) \\
& =\tau\left(x, x_{0}\right) \overline{\alpha(s x)} g\left(s, x x_{0}\right) .
\end{aligned}
$$

On $\bigoplus_{x \in \mathfrak{g}} L^{2}(S, \mu) \cong L^{2}(S \times g, \mu \times h)$, let $C g(s, x)=g\left(s, x^{-1}\right)$.

$C$ is a linear involutive isometry and

$$
C J_{\sigma} T_{f} J_{\sigma} C g(s, x)=\tau\left(x^{-1}, x_{0}\right) \overline{\alpha\left(s x^{-1}\right)} g\left(s, x_{0}^{-1} x\right) .
$$

Zeller-Meier's crossed product for the action $x M_{r}(\alpha)=M_{r}(s \mapsto \alpha(s x))$ of $g$ on $\left\{M_{r}(\alpha): \alpha \in L^{\infty}(S, \mu)\right\}$ is the von Neumann algebra generated by the operators $U x_{0}$ and $\Psi\left(M_{r}(\alpha)\right)$ on $\bigoplus_{x \in \mathbb{g}} L^{2}(S, \mu)$ defined by

$$
\left[U x_{0}\right]_{x, y}=\delta_{x}^{x_{0} y} \tau\left(x^{-1}, x_{0}\right) \text { and }\left[\Psi\left(M_{r}(\alpha)\right)\right]_{x_{y}}=\delta_{x}^{y}\left(x^{-1} M_{r}(\alpha)\right) \text {. }
$$

Hence

$$
U x_{0} g(s, x)=\sum_{y \in \mathfrak{g}} \delta_{x}^{x_{0} y} \tau\left(x^{-1}, x_{0}\right) g(s, y)=\tau\left(x^{-1}, x_{0}\right) g\left(s, x_{0}^{-1} x\right)
$$

and

$$
\Psi\left(M_{r}(\alpha)\right) g(s, x)=\sum_{y \in \mathfrak{g}} \delta_{x}^{y}\left(\alpha\left(s x^{-1}\right) g(s, y)\right)=\alpha\left(s x^{-1}\right) g(s, x) .
$$


Comparing these equalities with (4.9), we find that $C J_{\sigma}\left\{T_{f}: f \in I I(S \times\right.$ g) $\}^{\prime \prime} J_{\mathrm{\sigma}} C$ coincides with the crossed product. Since $L^{\prime}=J L^{\prime \prime} J=J K\left\{T_{f}\right.$ : $f \in I I(S \times g)\}^{\prime \prime} K^{-1} J=K J_{\sigma}\left\{T_{f}: f \in I I(S \times g)\right\}^{\prime \prime} J_{\sigma} K^{-1}, L^{\prime}$ is spacially isomorphic to the crossed product.

A useful extension of the unicity statement of Theorem 4.3 is the following lemma, treating two strictly equivalent strict cocycles $\sigma$ and $\omega$.

LEMMA 4.10. Suppose $\gamma: G \rightarrow T$ is a Borel function such that $\omega(x, y)=$ $\gamma(x y) \gamma(x)^{-1} \gamma(y)^{-1} \sigma(x, y)$ for all $(x, y) \in G^{(2)} . X \mapsto \gamma X$ is a one-one map from the set of $\sigma$-representations of $G$ onto the set of $\omega$-representations. $X^{\prime}$ and $(\gamma X)^{\prime}$ coincide. The commuting algebras of the $\sigma$-regular and $\omega$-regular representations are spacially isomorphic via a decomposable isometry intertwining the modular involutions.

Proof. Suppose $G$ is homogeneous. Define $\Gamma: L^{2}(G, \nu) \stackrel{\sim}{\rightarrow} L^{2}(G, \nu)$ by $\Gamma f(y)=\gamma\left(y^{-1}\right)^{-1} f(y)$.

$$
\begin{aligned}
\left(\gamma W_{\sigma}\right)(x) \Gamma f(y) & =\gamma(x) \sigma\left(y^{-1}, x\right)^{-1} \gamma\left(\left(x^{-1}\right)^{-1}\right)^{-1} f\left(y^{-1} x\right) \\
& =\gamma\left(y^{-1}\right)^{-1}\left(W_{\omega}(x) f\right)(y)=\left(\Gamma W_{\omega}(x) f\right)(y) .
\end{aligned}
$$

Let $J_{\sigma}$ be defined by (4.6), $J_{\omega}$ the operator constructed correspondingly using $\omega$. Then a simple calculation shows that $\Gamma J_{\omega}=J_{\sigma} \Gamma$. From this follows the spacial isomorphism of the regular representations if $G$ is homogeneous. For the general case, $G$ can be decomposed as the disjoint union of homogeneous groupoids. The equivalence is constructed on each summand as in the proof of Theorem 4.3.

REMARK 4.11. As defined in $\S 10$ of [20], not necessarily strict cocycles and equivalences can be made strict by passage to an i.r. In this way the word "strict" can be removed from all of the hypotheses in $\$ 4$.

5. The regular representation of principal groupoids. The structure of the regular representation of an ergodic groupoid $G$ is influenced both by the nature of the isotropy groups $G \mid\{u\}$ and by the structure of the ergodic equivalence relation defined by $G$. In one extreme case, there is only one unit, so that the groupoid is actually (defined by) a separable locally compact group. At the opposite extreme, there is no isotropy at all, so that the groupoid is principal and can be identified with the ergodic equivalence relation which it defines. In the case of a principal groupoid, much of the structure of the associated von Neumann algebra can be related directly to the structure of the groupoid itself. In the present section we make this analysis.

We retain the conventions and notation of $\S 4$. Again $\sigma$ is a fixed strict 
2-cocycle. $L$ refers to the subrepresentation of the regular representation of $G^{\sigma}$ discussed in Theorem 4.3.

THEOREM 5.1. Suppose $G$ is principal. The algebra $\mathcal{Z}$ of multiplication operators $M_{r}(\alpha)=(\tilde{g} \mapsto \tilde{g} \alpha \circ r)$ for $\alpha \in L^{\infty}\left(U_{G}, \mu\right)$ is a maximal abelian subalgebra of $L^{\prime \prime} . L^{\prime \prime}$ is a factor iff $G$ is ergodic. $L^{\prime \prime}$ is semifinite iff there is a positive Borel function $D$ on $U_{G}$ such that $\Delta=D \circ r / D \circ d$ a.e.

PRoof. First we characterize the center of $L^{\prime \prime} . J \mathscr{Z} J$ is multiplications $M_{d}(\alpha)$ by $\alpha \circ d$ for $\alpha \in L^{\infty}\left(U_{G}, \mu\right)$. ( $\left.\mathscr{Z} \cup J \mathscr{Z} J\right)^{\prime \prime}$ is the algebra of multiplications $M(k)$ by $k$ for $k \in L^{\infty}(G, v)$. Since $\mathscr{Z} \subset L^{\prime \prime}$ by Proposition 4.1, $J \mathscr{Z} J \subset L^{\prime}$ by Theorem 4.3. Since $K^{-1}(\mathscr{Z} \cup J \mathscr{Z} J)^{\prime \prime} K$ is maximal abelian in the algebra of all bounded operators on $L^{2}(G, \nu),(\mathscr{L} \cup J \mathscr{Z} J)^{\prime \prime}$ is maximal abelian in $\left(L^{\prime \prime} \cup L^{\prime}\right)^{\prime \prime}$. Thus, any central element $T$ must be multiplication by $k \in$ $L^{\infty}(G, v)$.

Since $T$ commutes with $L$, (4.5) shows that $k(y)=k\left(x^{-1} y\right) \nu^{r(x)}$-a.e. for $\nu$-a.a. $x$. By Remark 3.10 in [12], $T=M(k)=M_{d}(\beta)$ for some $\beta \in$ $L^{\infty}\left(U_{G}, \mu\right)$. This shows

$$
(\mathscr{Q} \cup J \mathscr{J})^{\prime \prime} \cap L^{\prime}=J \mathscr{Z}
$$

Since $T=J M_{r}(\beta) J$ is central and $J L^{\prime \prime} J=L^{\prime}, M_{r}(\beta)$ belongs to $L^{\prime}$. Thus $\beta(r(y))=\beta\left(r\left(x^{-1} y\right)\right) \nu^{r(x)}$-a.e. for $\nu$-a.a. $x$, so $\beta \circ r=\beta \circ d \nu$-a.e. Conversely, any element $\alpha \in L^{\infty}\left(U_{G}, \mu\right)$ satisfying $\alpha \circ r=\alpha \circ d \nu$-a.e. defines an element $M_{r}(\alpha) \in \mathscr{Z} \cap J \mathscr{Z}$, which is therefore central. It follows that $L^{\prime \prime}$ is a factor iff $G$ is ergodic.

By (5.2), $(\mathscr{Z} \cup J \mathscr{Z} J)^{\prime \prime} \cap L^{\prime \prime}=\mathscr{Z}$. Thus $\mathscr{Z}$ in $L^{\prime \prime}$ is maximal abelian: $\mathscr{Z}^{\prime} \cap L^{\prime \prime} \subset(\mathscr{Z} \cup J \mathscr{Z} J)^{\prime \prime} \cap L^{\prime \prime}=\mathscr{Z}$.

Suppose now that $L^{\prime \prime}$ is semifinite. By Theorem 14.2 of [27], there is a unitary representation $U: \mathbf{R} \rightarrow L^{\prime \prime}$ such that for all $\tilde{f} \in I I\left(G^{\sigma}\right)$, $U(t) L_{\tilde{f}} U(-t)=\Delta^{i t} L_{\tilde{f}} \Delta^{-i t}$. Here $\Delta^{i t}$ denotes the bounded operator $M\left(\Delta^{i t}\right)$ on $B L^{2}\left(G^{\sigma}, \nu \times m\right)$. Let $V(t)=\Delta^{i t} U(-t)$. The following calculation shows that $V(t) \in L^{\prime}$ for all $t \in \mathbf{R}$. $V(t) L_{\tilde{f}}=\Delta^{i t} U(-t) L_{\tilde{f}} U(t) U(-t)=$ $\Delta^{i t} \Delta^{-i t} L_{j} \Delta^{i t} U(-t)=L_{f} V(t)$. Since $\Delta^{i t} \in(\mathscr{Z} \cup J \mathscr{Z} J)^{\prime \prime} \subset(\mathscr{Z} \cup J \mathscr{L})^{\prime}, U(t)$ $\in \mathscr{Z}^{\prime} \cap L^{\prime \prime} \subset \mathscr{Z}$ and $V(t) \in(\mathscr{Z} \cup J \mathscr{Z} J)^{\prime \prime} \cap L^{\prime}=J \mathscr{Z}$. Since $V\left(t_{1}+t_{2}\right)=$ $V\left(t_{1}\right) V\left(t_{2}\right)$, we may apply Stone's Theorem to the unitary representations $U$ and $V$ to obtain real-valued Borel functions $a$ and $b$ on $U_{G}$ such that $U(t)$ is multiplication by $e^{i t a \circ r}$ and $V(-t)$ is multiplication by $e^{i t b o d}$. Let $\alpha=e^{i t a}$ and $\beta=e^{i t b}$. Since $\Delta^{i t}=V(t) U(t), \Delta^{i t}=\alpha \circ r / \beta \circ d \lambda$-a.e. From this we calculate

$$
\begin{aligned}
& 0=\iint\left|\Delta^{i t}(x)-\alpha(r(x)) / \beta(d(x))\right| d \lambda_{r(y)}(x) d \lambda(y), \\
& 0=\iint\left|\Delta^{i t}\left(x y^{-1}\right)-\alpha(r(x)) / \beta(r(y))\right| d \lambda_{d(y)}(x) d \lambda(y),
\end{aligned}
$$




$$
\begin{aligned}
& 0=\iint\left|\Delta^{i t}(x) \Delta^{i t}\left(y^{-1}\right)-\alpha(r(x)) / \beta(r(y))\right| d \lambda_{d(y)}(x) d \lambda(y), \\
& 0=\iint\left|\frac{\alpha(r(x))}{\beta(d(x))} \frac{\alpha(d(y))}{\beta(r(y))}-\frac{\alpha(r(x))}{\beta(r(y))}\right| d \lambda_{d(y)}(x) d \lambda(y), \\
& 0=\iint|\alpha(d(y))-\beta(d(x))| d \lambda_{d(y)}(x) d \lambda(y), \\
& 0=\iint|\alpha(d(y))-\beta(d(y))| d \lambda(y) .
\end{aligned}
$$

Thus $e^{i t a}=e^{i t b} \tilde{\lambda}$-a.e., so $\Delta^{i t}=\exp (i t(a \circ r-a \circ d))$ a.e. Comparing infinitesimal generators, $\ln \Delta(x)=a \circ r(x)-a \circ d(x)$ for $\lambda$-a.a. $x$. Thus $\Delta(x)=$ $\exp (a \circ r(x)) / \exp (a \circ d(x))$ a.e.

Suppose conversely that $\Delta=D \circ r / D \circ d$ a.e. Then $\Delta^{i t} L_{\tilde{f}} \Delta^{-i t}=$ $M_{r}\left(e^{i t D}\right) L_{\tilde{f}} M_{r}\left(e^{-i t D}\right)$ since $M_{d}\left(e^{i t D}\right)$ commutes with $L$. Thus the automorphism $L_{\dot{f}} \rightarrow \Delta^{i t} L_{j} \Delta^{-i t}$ of $L^{\prime \prime}$ is inner. By Theorem 14.1 of [27], $L^{\prime \prime}$ is semifinite.

The "if" part of the semifiniteness condition does not require $G$ to be principal.

A homomorphism $D \circ r / D \circ d$ on an i.r. is called similar to $1[20, \S 6]$. The proof of Theorem 5.1 shows that the automorphism $L_{\tilde{f}} \mapsto \Delta^{i t} L_{\tilde{f}} \Delta^{-i t}$ is inner iff the homomorphism $x \mapsto \Delta^{i t}(x)$ is similar to 1 . Also, this terminology suggests that the condition for semifiniteness can be sharpened:

COROLlaRY 5.3. For a principal measure groupoid, the following are equivalent:

(1) The commuting algebra of the regular representation is semifinite.

(2) For some strict cocycle $\sigma$, the commuting algebra of the o-regular representation is semifinite.

(3) For every strict cocycle $\sigma$, the commuting algebra of the $\sigma$-regular representation is semifinite.

(4) $(\mathrm{g}, C)$ has a Haar measure whose modular homomorphism is 1.

Proof. The first three conditions all are equivalent by Theorem 5.1 to the condition that the modular homomorphism of some (and hence every) Haar measure is similar to the identity. The fourth condition implies this, so it suffices to produce a Haar measure with modular function 1 from a Haar measure $(\nu, \mu)$ with modular function $D \circ r / D \circ d$ a.e. This does not require $g$ to be principal. Let $\nu_{1}$ be defined by $\nu_{1}(E)=\iint 1_{E} D \circ d d \nu^{u} d \mu(u) .\left(\nu_{1}, \mu\right)$ is a Haar measure and $\int f\left(x^{-1}\right) d \nu_{1}(x)=\int f\left(x^{-1}\right) D(d(x)) d \nu(x)=$ $\int f(x) D(r(x)) \Delta(x)^{-1} d \nu(x)=\int f(x) D(d(x)) d \nu(x)=\int f(x) d \nu_{1}(x)$. Thus 1 is the modular homomorphism of $\left(\nu_{1}, \mu\right)$.

Since a transitive principal groupoid is similar to the group $\{0\}$, it follows 
from Remark 4.7 that for such a groupoid $W^{\prime}$ is type I and from discussion in $\S 10$ of [20] that every cocycle is equivalent to 1 . By Lemma 4.10 , for each cocycle $\sigma,\left(W^{\sigma}\right)^{\prime}$ is type $I$. It is significant that the converse holds.

THEOREM 5.4. Let $(G, C)$ be a principal ergodic groupoid, $\sigma$ a 2-cocycle. If $\left(W^{\sigma}\right)^{\prime}$ is type $\mathrm{I}$, then $G$ is essentially transitive.

Proof. We may assume that $\mu=\tilde{\lambda}$ and that $\sigma$ is strict. We adopt notation of Example 3.11. Since $\left(W^{\sigma}\right)^{\prime}$ is a factor, there is an isomorphism $\Phi$ of $\left(W^{\sigma}\right)^{\prime}$ onto the algebra of all bounded operators on a Hilbert space $K$. Let $\mathcal{L} C(K)$ be the $C^{*}$-algebra of compact operators. $\pi=\Phi^{-1} \mid \mathcal{L C}(K)$ is a factor representation of $\mathcal{L} C(K)$ whose image generates $\left(W^{\sigma}\right)^{\prime}$. Since the image of $\pi$ commutes with the algebra $\mathcal{Z}$ of multiplications $M_{r}(\alpha)\left(\alpha \in L^{\infty}\left(U_{G}, \tilde{\lambda}\right)\right), \pi$ may be decomposed $\pi=\int \pi_{u} d \tilde{\lambda}(u)$ on $\int H d \tilde{\lambda}$, [6, Lemme 8.3.1]. Since $\mathscr{Z}$ is maximal abelian in $\left(W^{\sigma}\right)^{\prime \prime}$ by Theorems 4.1 and 5.3, a.a. $\pi_{u}$ are irreducible [6, Lemme 8.5.1]. Hence by [6, Corollaire 4.15], a.a. $\pi_{u}$ are unitarily equivalent; by Proposition 8.17 of [6] there is a decomposable isometry $V_{1}=$ $\int V_{1}(u) d \tilde{\lambda}(u)$ of $\int H d \tilde{\lambda}(u)$ onto $\int K d \tilde{\lambda}(u)$ such that for a.a. $u V_{1}(u) \pi_{u} V_{1}(u)^{-1}$ is the identity representation of $\operatorname{CC}(K)$.

Let $A_{1}, A_{2}, \ldots$ be a dense subsequence in $\mathcal{L C}(K)$. For each $i$ there is an i.r. $G \mid U_{i}$ such that $x \in G \mid U_{i}$ implies $\pi_{r(x)}\left(A_{i}\right) W^{\sigma}(x)=W^{\sigma}(x) \pi_{d(x)}\left(A_{i}\right)$ and $u \in U_{i}$ implies $V_{1}(u) \pi_{u} V_{1}(u)^{-1}=$ identity and $V_{1}(u)$ is an isometry. If $x \in$ $G \mid\left(\cap U_{i}\right)$, then

$$
\begin{aligned}
A_{i} V_{1}( & r(x)) W^{\sigma}(x) V_{1}(d(x))^{-1} \\
& =V_{1}(r(x)) \pi_{r(x)}\left(A_{i}\right) V_{1}(r(x))^{-1} V_{1}(r(x)) W^{\sigma}(x) V_{1}(d(x))^{-1} \\
& =V_{1}(r(x)) W^{\sigma}(x) \pi_{d(x)}\left(A_{i}\right) V_{1}(d(x))^{-1} \\
& =V_{1}(r(x)) W^{\sigma}(x) V_{1}(d(x))^{-1} A_{i} .
\end{aligned}
$$

Thus there is a Borel function $c: G \rightarrow \mathbf{T}$ such that a.e. $V_{1}(r(x)) W^{\sigma}(x) V_{1}(d(x))^{-1}=c(x) I$. Consequently, by choosing $V$ differently in Example 3.11, we may assume $W^{\sigma}(x)=c(x) I$ for all $x$ in an i.r. Since then on an i.r. $c(x y)=\sigma(x, y) c(x) c(y)$, by Lemma 4.10 we may assume $\sigma=1$.

Since the abelian algebra $\mathscr{Z}_{d}$ of multiplications $M_{d}(\alpha)\left(\alpha \in L^{\infty}\left(U_{G}, \tilde{\lambda}\right)\right)$ commutes with $W_{1}$, the image of $\mathscr{Z}_{d}$ under $T \mapsto V T V^{-1}$ is an abelian algebra of the form $\mathbf{C I} \otimes \mathbb{Q}$ under the isomorphism $L^{2}\left(U_{G}, \tilde{\lambda}, H\right) \approx L^{2}\left(U_{G}, \tilde{\lambda}\right) \otimes H$. Since $G$ is principal, the multiplications $M_{r}(\alpha)\left(\alpha \in L^{\infty}\left(U_{G}, \tilde{\lambda}\right)\right)$, together with $\mathbf{C I} \otimes \mathbb{Q}$, generate a maximal abelian algebra on $L^{2}\left(U_{G}, \tilde{\lambda}\right) \otimes H$, so we can arrange that $H=L^{2}(\mathbf{R}, \eta)$, where $\eta$ is a probability, and that $\mathbb{Q}$ is multiplications by elements of $L^{\infty}(\mathbf{R}, \eta)$.

$V$ induces a Boolean isomorphism of $L^{\infty}(G, \nu)$ with $L^{\infty}\left(U_{G} \times \mathbf{R}, \lambda \times \eta\right)$; 
since these analytic spaces are metrically standard, by Theorem 2.1 of [20] there is a Borel isomorphism $\gamma$ from a.a. $U_{G} \times \mathbf{R}$ onto a.a. $G$ realizing the Boolean isomorphism. Let $\rho=\left(d \nu / d \gamma_{*}(\tilde{\lambda} \times \eta)\right)^{1 / 2} \circ \gamma . f \mapsto(f \circ \gamma) \rho$ is an isomorphism $\tilde{V}$ of $L^{2}(G, \nu)$ with $L^{2}\left(U_{G} \times \mathbf{R}, \tilde{\lambda} \times \eta\right)$ such that $V \tilde{V}^{-1}$ commutes with the maximal abelian algebra representing $L^{\infty}\left(U_{G} \times \mathbf{R}, \tilde{\lambda} \times\right.$ $\eta)$. Thus there is a Borel function $k: U_{G} \times \mathbf{R} \rightarrow \mathbf{T}$ such that $V \tilde{V}^{-1}=M(k)$, multiplication by $k$. Hence also for a.e. $u V(u) \tilde{V}(u)^{-1}=M(k)$. Since for a.a. $u \quad \tilde{V}(u) f(u, t)=f(\gamma(u, t)) \rho(u, t)$, we have for a.a. $u V(u) f(u, t)=$ $f(\gamma(u, t)) \rho(u, t) k(u, t) \eta$-a.a.

By passage to an i.r., we may assume that all $V(u) f(u, t)=$ $f(\gamma(u, t)) \rho(u, t) k(u, t) \eta$-a.e., that all $V(u)$ are isomorphisms, and that $W=$ $c I$ is a strict representation. Define a measure $\eta^{u}$ on $G$ by

$$
\begin{aligned}
\int f(y) d \eta^{u}(y) & =(V(u) f, k) \\
& =\int f(\gamma(u, t)) \rho(u, t) k(u, t) \overline{k(u, t)} d \eta(t) \\
& =\int f(\gamma(u, t)) \rho(u, t) d \eta(t) .
\end{aligned}
$$

We calculate $\int f\left(x^{-1} y\right) d \eta^{r(x)}(y)=\left(V(r(x)) W_{1}(x) f, k\right)=c(x)(V(d(x)) f, k)$ $=c(x) \int f(y) d \eta^{d(x)}(y)$. Thus $c(x)=1$ and $x^{-1} \eta^{r(x)}=\eta^{d(x)}$. Furthermore,

$$
\begin{aligned}
\int f(y) d \nu^{u}(y) & =\int \sqrt{f(y)}^{2} d \nu^{u}(y)=\int|V(u) \sqrt{f}|^{2} d \eta \\
& =\int f(\gamma(u, t)) \rho(u, t)^{2} d \eta(t) \\
& =\int f(\gamma(u, t)) \rho \circ \gamma^{-1}(\gamma(u, t)) \rho(u, t) d \eta(t) \\
& =\int f(y) \rho\left(\gamma^{-1}(y)\right) d \eta^{u}(y),
\end{aligned}
$$

so the Radon-Nikodým derivative of $\int \eta^{u} d \tilde{\lambda}(u)$ with respect to $\nu$ is $\left(\rho \circ \gamma^{-1}\right)^{-1}$.

By Theorem 3.9 of [12], there exists a Borel function $\phi: U_{G} \rightarrow \mathbf{R}_{+}^{*}$ such that $\phi(d(y))=\rho\left(\alpha^{-1}(y)\right)^{-1}$ for $\nu^{u}$-a.a. $y$ for $\tilde{\lambda}$-a.a. u. $\int f(y) \phi \circ d(y) d \eta^{u}(y)=$ $\int f(\gamma(u, t)) \rho(u, t)^{-1} \rho(u, t) d \eta(t)=\int f(\gamma(u, t)) d \eta(t)$. Since $\eta(\mathbf{R})=1, E \mapsto$ $\int 1_{E} \phi \circ d d \eta$ is a finite Haar measure. By Theorem 5.1 of [12], $G$ is essentially transitive.

Let $(G,[\lambda])$ be an ergodic groupoid, $\Psi: G \rightarrow \mathbf{R}_{+}^{*}$ a strict homomorphism. The closure of the range of $\Psi$ (see $[20, \S 7]$ ) is the $\mathbf{R}_{+}^{*}$-action defined as follows: Let $\eta^{*}$ be Lebesgue measure on $\mathbf{R}_{+}^{*}$. The subalgebra $\left\{g \in L^{\infty}\left(U_{G} \times\right.\right.$ $\left.\mathbf{R}_{+}^{*}, \tilde{\lambda} \times \eta^{*}\right): g(r(x), a)=g(d(x), a \Psi(x))$ for $\lambda \times \eta^{*}$-a.a. $\left.(x, a)\right\}$ is stable under the $\mathbf{R}_{+}^{*}$-action $\left(T_{b} f\right)(u, a)=g\left(u, b^{-1} a\right)$. This action induces an ergodic 
$\mathbf{R}_{+}^{*}$-action on an analytic probability space. The point action so constructed is the range closure of $\Psi$. Up to metric isomorphism, the range closure depends only on the similarity class of $\Psi$.

To any properly infinite von Neumann algebra with separable predual, Connes and Takesaki have associated an $\mathbf{R}_{+}^{*}$-action which they call the smooth flow of weights [3, Definition $\operatorname{II}(2.6)]$. Let $(G, C)$ be a principal ergodic groupoid with Haar measure $(\nu, \tilde{\lambda})$ and modular homomorphism $\Delta$ not similar to the identity. Let $\sigma$ be a strict cocycle, $L$ the $\sigma$-regular representation as in Theorem 4.3. $L^{\prime \prime}$ is purely infinite.

THEOREM 5.5. The smooth flow of weights of $L^{\prime \prime}$ is metrically isomorphic to the range closure of $\Delta$.

The proof was obtained after helpful discussion with A. Ramsay.

Proof. For convenience, write $L[\tilde{f}]$ for the operator, previously denoted $L_{\tilde{f}}$, associated to the function $\tilde{f}$ by the $\sigma$-regular representation. $L^{\prime \prime}$ is generated by the modular Hilbert algebra $B$ था $_{I}\left(G^{\sigma}\right)$ on $B L^{2}\left(G^{\sigma}, \nu \times m\right)$. Let $\phi$ be the associated weight. Multiplication by $\Delta$ is the modular operator; and Ad $\Delta^{i a}=\left(L[\tilde{f}] \mapsto \Delta^{i a} L[\tilde{f}] \Delta^{-i a}\right)$ is the modular automorphism group $\sigma_{a}^{\phi}(a \in$ R).

Now we establish some notation. Let $a \mapsto U_{a}$ be the regular representation of $\mathbf{R}$, acting on $L^{2}(\mathbf{R}, \eta)$, where $\eta$ is Lebesgue measure. Let $F$ be the Fourier transform and define $V_{a}=F U_{a} F^{-1}$. Let $e_{a}(b)=\exp (i a b)$. The formulae $U\left(e_{a}\right)=U_{a}$ and $V\left(e_{a}\right)=V_{a}$ define by extension injective homomorphisms of $L^{\infty}(\mathbf{R})$ into the algebra $\mathcal{L}\left(L^{2}(\mathbf{R})\right)$ of bounded operators on $L^{2}(\mathbf{R})$. $U_{a}=\rho^{i a}$ with $\rho$ a positive selfadjoint operator. Let $\omega$ be the weight $T \mapsto \operatorname{Tr}(\rho T)$ on $\mathcal{L}\left(L^{2}(\mathbf{R})\right) \cdot \sigma_{a}^{\omega}=\operatorname{Ad} U_{a}$.

Regard the Hilbert space $B L^{2}\left(G^{0}, \nu \times m\right) \otimes \mathcal{L}\left(L^{2}(\mathbf{R})\right)$ as $L^{2}\left(\mathbf{R}, \eta, B L^{2}(G, \nu \times m)\right) . L^{\prime \prime} \otimes \mathfrak{L}\left(L^{2}(\mathbf{R})\right)$ acts on this space; and $\bar{\omega}=\phi \otimes \omega$ is a weight on $L^{\prime \prime} \otimes \mathcal{L}\left(L^{2}(\mathbf{R})\right)$. Let $N_{\bar{\omega}}$ be the von Neumann subalgebra generated by $I \otimes U_{a}(a \in \mathbf{R})$ and the direct integral operator $\bar{\pi}(T)=$ $\int\left(b \mapsto \sigma_{-b}^{\phi}(T)\right) d \eta(b)\left(T \in L^{\prime \prime}, b \in \mathbf{R}\right)$ in $L^{\prime \prime} \otimes V\left(L^{\infty}(\mathbf{R})\right)$. The action $\theta$ of $\mathbf{R}$ on $N_{\bar{\omega}}$ given by $\theta_{c}(S)=\left(I \otimes V_{c}\right) S\left(I \otimes V_{c}\right)^{*}\left(S \in N_{\bar{\omega}}, c \in \mathbf{R}\right)$ restricts to the center $C_{\bar{\omega}}$ of $N_{\bar{\omega}}$, inducing a point action $\theta$ of $\mathbf{R}$ such that $c \mapsto \theta_{(-\ln c)}$ is isomorphic to the smooth flow of weights [3, Lemma II.14 and Corollary II.2.5].

Let $\mathcal{Z}$ be the maximal abelian subalgebra $\left\{M_{r}(\alpha) \in L^{\prime \prime}: \alpha \in L^{\infty}\left(U_{G}, \tilde{\lambda}\right)\right\}$ as in Theorem 5.1. Since $\sigma_{t}^{\phi}\left(M_{r}(\alpha)\right)=M_{r}(\alpha)$ and $M_{r}(\alpha) \otimes U_{a}=\int(b \mapsto$ $\left.\boldsymbol{\sigma}_{-b}^{\phi}\left(M_{r}(\alpha)\right)\right) d \eta(b)\left(I \otimes U_{a}\right) \in N_{\bar{\omega}}, \mathcal{Z} \otimes U\left(L^{\infty}(\mathbf{R})\right)$ is a subset of $N_{\bar{\omega}}$.

Since $N_{\bar{\omega}} \subset L^{\prime \prime} \otimes \mathfrak{L}\left(L^{2}(\mathbf{R})\right)$ and $\mathscr{Z} \otimes U\left(L^{\infty}(\mathbf{R})\right)$ is maximal abelian in $L^{\prime \prime} \otimes \mathscr{L}\left(L^{2}(\mathbf{R})\right), C_{\bar{\omega}}$ is contained in $\mathscr{L} \otimes U\left(L^{\infty}(\mathbf{R})\right)$. Since any element of $\mathscr{Z} \otimes U\left(L^{\infty}(\mathbf{R})\right)$ commutes with all $I \otimes U_{a}$, an element $(u, a) \mapsto g(u, a)$ of 
$L^{\infty}\left(U_{G} \times \mathbf{R}, \lambda \times \eta\right)$ is such that $M_{r} \otimes U(g) \in C_{\bar{\omega}}$ iff $\bar{\pi}(L[\tilde{f}]) M_{r} \otimes U(g)=$ $M_{r} \otimes U(g) \bar{\pi}(L[\tilde{f}])$ for all $\tilde{f} \in \mathcal{U}_{I}\left(G^{\sigma}\right)$.

Suppose $g(u, c)=\alpha(u) e_{b}(c)$. Then

$$
\begin{gathered}
\left(\bar{\pi}(L[\tilde{f}])\left(M_{r} \otimes U\right)(g) k\right)(c)= \\
=L\left[\Delta^{-i c} L[\tilde{f}] \Delta^{i c}\right) M_{r}(\alpha)(k(b+c)) \\
\left(\left(M_{r} \otimes U\right)(g) \bar{\pi}(L[\tilde{f}]) k\right)(c)=M_{r}(\alpha)\left(\Delta^{-i c}\right](k(b+c)), \\
=L\left[\tilde{f} \alpha \circ r \Delta^{-i(b+c)}\right](k(b+c)) .
\end{gathered}
$$

If $g \in L^{\infty}\left(U_{G} \times \mathbf{R}, \tilde{\lambda} \times \eta\right)$ there is a sequence $g_{n} \in L^{\infty}\left(U_{G} \times \mathbf{R}, \tilde{\lambda} \times \eta\right)$ with $\left\|g_{n}\right\|_{\infty}$ bounded, $g_{n}(u, a)=\sum_{j=1}^{l(n)} \alpha_{j, n}(u) e_{b(j, n)}(a)$, such that $g_{n} \mapsto g$ a.e. and $M_{r} \otimes V\left(g_{n}\right) \rightarrow M_{r} \otimes V(g)$ strongly. Suppose

$$
g(r(x), c)=g(d(x), c+\ln \Delta(x)) \text { a.e. }
$$

Let $\tilde{f} \in \mathcal{Q}_{I}\left(G^{\sigma}\right)$ and $f(x)=\int s \tilde{f}(x, s) d m(s)$. The isometry $K: L^{2}(G, v) \rightarrow$ $L^{2}\left(G^{\sigma}, \nu \times m\right)$ was introduced for the proof of Theorem 4.3. $K \otimes I: L^{2}(G \times$ $\mathbf{R}, \nu \times \eta) \rightarrow L^{2}(G \times \mathbf{T} \times \mathbf{R}, \nu \times m \times \eta)$ is defined by

$$
(K \otimes I) h(x, s, a)=s^{-1} \sigma\left(x^{-1}, x\right) h(x, a) .
$$

Let $h_{1}, h_{2} \in L^{2}(G \times \mathbf{R}, \nu \times \eta)$.

$\left(\bar{\pi}(L[\tilde{f}]) M_{r} \otimes U(g)(K \otimes I) h_{1}\right.$

$\left.-M_{r} \otimes U(g) \bar{\pi}(L[\tilde{f}])(K \otimes I) h_{1},(K \otimes I) h_{2}\right)$

$$
\begin{gathered}
=\lim _{n \rightarrow \infty} \sum_{j=1}^{l(n)} \int\left(L\left[\tilde{f} \Delta^{-i(c+b(j, n))}\left(\left(\alpha_{j, n} \circ d\right) \Delta^{i b(j, n)}-\left(\alpha_{j, n} \circ r\right)\right)\right]\right. \\
\left.\cdot(K \otimes I) h_{1}(\cdot, c+b(j, n)), h_{2}(\cdot, c)\right) d \eta(c) \\
=\lim _{n \rightarrow \infty} \sum_{j=1}^{l(n)} \iiint f(x)\left(\alpha_{j, n}(d(x)) \Delta(x)^{i b(j, n)}-\alpha_{j, n}(r(x))\right) \sigma\left(y^{-1}, x\right)^{-1} \\
\cdot \Delta(x)^{-i(c+b(j, n))} h_{1}\left(x^{-1} y, c+b(j, n)\right) \overline{h_{2}(y, c)} \\
\cdot d \nu^{r(y)}(x) d \nu(y) d \eta(c) \\
=\lim _{n \rightarrow \infty} \sum_{j=1}^{l(n)} \iint f(x)\left(\alpha_{j, n}(d(x)) \Delta(x)^{i b(j, n)}-\alpha_{j, n}(r(x))\right) \sigma\left(y^{-1}, x\right)^{-1} \\
\cdot \int e_{b(j, n)}(c) F\left(a \mapsto h_{1}\left(x^{-1} y, a\right) \Delta(x)^{-i a}\right)(c) \\
\cdot \frac{F\left(a \mapsto h_{2}(y, a)\right)(c)}{F(\eta)} d \eta d \nu^{r(x)}(y) d \nu(x)
\end{gathered}
$$




$$
\begin{array}{r}
=\lim _{n \rightarrow \infty} \iiint f(x)\left(g_{n}(d(x), c+\ln \Delta(x))-g_{n}(r(x), c)\right) \sigma\left(y^{-1}, x\right)^{-1} \\
\cdot F\left(a \mapsto h_{1}\left(x^{-1} y, a\right) \Delta(x)^{-i a}\right)(c) \overline{F\left(a \mapsto h_{2}(y, a)\right)(c)} \\
\cdot d \eta(c) d \nu^{r(x)}(y) d \nu(x)=0
\end{array}
$$

by (5.6). From this it follows that (5.6) is sufficient for $M_{r} \otimes U(g)$ to belong to $C_{\bar{\omega}}$.

Conversely, suppose $M_{r} \otimes U(g) \in C_{\bar{\omega}}$. Let $\tilde{f} \in \mathcal{U}_{I}\left(G^{\sigma}\right)$ and $G(c)(x)=$ $g(d(x), c+\ln \Delta(x))-g(r(x), c)$. Using properties of the Fourier transform and the foregoing calculation, it is not difficult to show that for $\eta$-a.a. $c$, $L[\tilde{f} G(c)]=0 . \tilde{f} G(c) \in \mathcal{Q}_{I}\left(G^{o}\right)$ a.e., so $\|B(\tilde{f} G(c))\|_{2}=0$ a.e. by Theorem 4.3 and (4.4). Let $f \in \mathcal{Q}_{I}(G)$ and let $\tilde{f}(x, s)=s^{-1} f(x)$. Then $\|B(\tilde{f} G(c))\|_{2}=0$ implies $\int|f(x) G(c)(x)|^{2} d v(x)=0$. Thus for a.a. $c, G(c)=0$ a.e. by Lemma 2.6. Consequently, $g$ satisfies (5.6). The restriction of $M_{r} \otimes U$ is an isomorphism of the algebra satisfying (5.6) onto $C_{\bar{\omega}}$.

Let $\Gamma_{a}$ be the automorphism of $L^{\infty}\left(U_{G} \times \mathbf{R}, \tilde{\lambda} \times \eta\right)$ defined for $g(u, c)=$ $\alpha(u) e_{b}(c)$ by $\left(\Gamma_{a} g\right)(u, c)=\alpha(u) e_{b}(a+c)$.

$$
\begin{aligned}
\left(I \otimes V_{a}\right) M_{r} \otimes U(g)\left(I \otimes V_{a}\right)^{*} k(c) & =e_{a}(c) M_{r}(\alpha)\left(I \otimes V_{a}\right)^{*} k(b+c) \\
& =e_{a}(c) M_{r}(\alpha) e_{-a}(b+c) k(b+c) \\
& =M_{r} \otimes U\left(\Gamma_{-a} g\right) k(c) .
\end{aligned}
$$

Thus, if $C_{\bar{\omega}}$ is isomorphic to $L^{\infty}(\Omega, p), a \mapsto \Gamma_{-a}$ induces on $\Omega$ a point action $\Gamma$ metrically isomorphic to that induced by $a \mapsto \hat{\theta}_{a}$. By the definition of the closure of the range of $\Delta, a \mapsto \Gamma_{\ln a}$ is metrically isomorphic to the range closure of $\Delta$. The result now follows from the characterization of the smooth flow of weights already given.

The possibility of studying the regular representation of measure groupoids has been mentioned by Connes [1]. Feldman and Moore ([8] and [9]) have studied independently of us the $\sigma$-regular representations of ergodic equivalence relations such that each equivalence class is countable. They show that every such relation is furnished by the action of a countable discrete group, although they do not know whether the group can be taken to be freely acting. The von Neumann algebras generated by the 1-regular representation of a principal groupoid furnished by the unfree action of a countable group were first described by Krieger [15].

For principal groupoids furnished by arbitrary separable locally compact group actions, Krieger's construction has been generalized independently of us by Samuelides and Sauvageot [25]. Sauvageot [26] has pointed out that the finiteness condition of [9] holds for arbitrary principal groupoids: for the regular representation to be finite, a.a. $\lambda^{u}$ must be discrete measures; and then the necessary and sufficient condition in [9] is applicable. Sauvageot also 
has calculated the flow of weights of the 1-regular representation, a special case of Theorem 5.5.

The map $L_{f} \mapsto L_{\bar{f}}$ is an anti-isomorphism of the 1-regular representation von Neumann algebra with itself. Connes has proved [2] that there is a principal ergodic groupoid $G$, furnished by a discrete group action, and a cocycle $\sigma$ of $G$, such that the $\sigma$-regular representation factor is not antiisomorphic to itself. Thus, there is no hope of obtaining all factors from the 1-regular representations of principal ergodic groupoids. It is not known whether every factor can be obtained from the $\sigma$-regular representations of principal ergodic groupoids; nor is it known whether it suffices to consider only principal groupoids furnished by group actions. Recent investigation in collaboration with Feldman and Moore has shown that every factor obtained from a $\sigma$-regular representation of a principal groupoid furnished by a group action can be obtained from a $\sigma$-regular representation of a principal groupoid furnished by the action of a discrete group. Details of this result will be reported elsewhere [7].

6. Ergodic decomposition of a measure groupoid. Let $(G,[\lambda])$ be a measure groupoid, $\left(\mathcal{E},\left[\lambda^{\prime}\right]\right)$ the associated principal groupoid. Let $(\nu, \tilde{\lambda})$ be a Haar measure, $P=d \nu / d \lambda$. The proof of Theorem 5.1 shows that the center of the regular representation von Neumann algebra of $\mathcal{E}$ is isomorphic to the subalgebra $\mathscr{Z}_{0}$ of $L^{\infty}\left(U_{G}, \tilde{\lambda}\right)$ consisting of functions $\alpha \in L^{\infty}\left(U_{G}, \tilde{C}\right)$ such that $\alpha \circ d=\alpha \circ r$ a.e. This is also isomorphic to the commuting algebra of the one-dimensional identity representation of $G$. By decomposing $L^{2}\left(U_{G}, \tilde{\lambda}\right)$ with respect to $\mathscr{Z}_{0}$, we obtain at once an ergodic decomposition of $G$, a central decomposition of the regular representation of $\mathcal{E}$, and an irreducible decomposition of the representation $f \mapsto I I_{f}$ of $I I(G)$ induced by the identity representation of $G$ (cf. Lemma 1.4). This theorem has also been obtained by Ramsay [23].

TheORem 6.1 (ERgodic Decomposition). Let $(G, C)$ be a measure groupoid, $\lambda \in C$ a symmetric quasi-invariant probability with $r$-decomposition $\lambda=\int \lambda^{u} d \tilde{\lambda}(u)$. There is an analytic probability space $(\Omega, \eta)$ and a Borel map $\pi$ from $U_{G}$ onto $\Omega$ such that $\eta=\pi_{*} \tilde{\lambda}=E \mapsto \tilde{\lambda}\left(\pi^{-1}(E)\right)$ and if $\tilde{\lambda}=\int \lambda_{\omega} d \eta(\omega)$ is the $\pi$-decomposition of $\tilde{\lambda}$, then a.a. $\left(G,\left[\int \lambda^{u} d \tilde{\lambda}_{\omega}(u)\right]\right)$ are ergodic groupoids. If $\Omega^{\prime}$ and $\pi^{\prime}$ have these properties, then there is a Borel isomorphism i from a.a. $\Omega$ onto a.a. $\Omega^{\prime}$ such that $\pi^{\prime}=i \circ \pi$ a.e.

Proof. Let $\mathcal{Q}$ be a suitably large separable algebra of $I(G)$, as in Remark 3.3. By Theorem 3.8, $M_{0}=M_{r}\left(\mathscr{L}_{0}\right)$ is a maximal abelian subalgebra of $\left\{I_{f}\right.$ : $f \in \mathbb{Q}\}^{\prime}$. Obtain $\Omega$ and $\pi$ by decomposing $L^{2}\left(U_{G}, \tilde{\lambda}\right)=\int L^{2}\left(U_{G}, \tilde{\lambda}_{\omega}\right) d \eta(\omega)$ and $I I_{f}=\int I I_{f}^{\omega} d \eta(\omega)$ as a direct integral of irreducibles. A computation 
shows that $\left(I I_{f}^{\omega} j\right)(u)=\int f(x) j(d(x)) d \nu^{u}(x)\left(j \in L^{2}\left(U_{G}, \tilde{\lambda}_{\omega}\right), f \in \mathbb{Q}\right)$ for $\eta$ a.a. $\omega$.

Let $\lambda_{\omega}=\int \lambda^{u} d \tilde{\lambda}_{\omega}(u)$. We show $\left(G, \lambda_{\omega}\right)$ are a.a. measure groupoids. If $F \in \mathscr{B}(\Omega)$ and $E \in \mathscr{B}(G)$, then

$$
\begin{aligned}
\int 1_{F}(\omega) \int 1_{E}(x) d \lambda_{\omega}(x) d \eta(\omega) & =\int 1_{F} \circ \pi(u) \int 1_{E}(x) d \lambda^{u}(x) d \lambda(u) \\
& =\int 1_{F} \circ \pi \circ r(x) 1_{E}(x) d \lambda(x) \\
& =\int 1_{F} \circ \pi \circ d(x) 1_{E}\left(x^{-1}\right) d \lambda(x) \\
& =\int 1_{F} \circ \pi \circ r(x) 1_{E}\left(x^{-1}\right) d \lambda(x) \\
& =\int 1_{F}(\omega) \int 1_{E}\left(x^{-1}\right) d \lambda_{\omega}(x) d \eta(\omega) .
\end{aligned}
$$

Since $\mathscr{B}(\Omega)$ is countably generated, a.a. $\lambda_{\omega}$ are symmetric. Quasi-invariance of a.a. $\lambda_{\omega}$ follows from the quasi-invariance of $\lambda$ and the $r$-decomposition $\lambda_{\omega}=\int \lambda^{u} d \tilde{\lambda}_{\omega}(u)$. The same argument shows that if $\nu_{\omega}(E)=$ $\int_{E} P d \lambda^{u} d \tilde{\lambda}_{\omega}(u)$, then for a.a. $\omega\left(\nu_{\omega}, \tilde{\lambda}_{\omega}\right)$ is a Haar measure for $\left(G,\left[\lambda_{\omega}\right]\right)$. Since for a.a. $\omega, I I^{\omega}$ is an irreducible representation of $\mathcal{Q}$, it follows from Theorem 3.8 that for a.a. $\omega$ the identity representation of $\left(G,\left[\lambda_{\omega}\right]\right)$ is irreducible. Thus a.a. $\left(G,\left[\lambda_{\omega}\right]\right)$ are ergodic.

To prove the uniqueness part of the theorem, it suffices to show that if $\Omega, \eta$, and $\pi$ satisfy the existence statement of the theorem, then $\mathscr{L}_{0}$ is the image of $L^{\infty}(\Omega, \eta)$ under the injection induced by $\pi$. Suppose $\alpha \in \mathscr{L}_{0}$. Then $\alpha \circ r=$ $\alpha \circ d \lambda$-a.e., so $\alpha \circ r=\alpha \circ d \lambda_{\omega}$-a.e., for $\eta$-a.a. $\omega$. Since a.a. $\left(G, \lambda_{\omega}\right)$ are ergodic, $\alpha(u)=\int \alpha(v) d \tilde{\lambda}_{\omega}(v)=\tilde{\alpha}(\omega) \tilde{\lambda}_{\omega}$-a.e. for $\eta$-a.a. $\omega . \tilde{\alpha} \in L^{\infty}(\Omega, \eta)$ and $\tilde{\alpha} \circ \pi=\alpha \tilde{\lambda}$-a.e. Conversely, if $\tilde{\beta} \in L^{\infty}(\Omega, \eta)$, then $\beta \circ \pi$ is $\tilde{\lambda}_{\omega}$-essentially constant for $\eta$-a.a. $\omega$, so $\tilde{\beta} \circ \pi \circ r=\tilde{\beta} \circ \pi \circ d \lambda_{\omega}$-a.e. for $\eta$-a.a. $\omega$. Hence $\beta \circ \pi \in \mathscr{L}_{0}$.

RemarK 6.2. Suppose $x \cdot \lambda^{d(x)} \sim \lambda^{r(x)}$ for all $x \in G$. By Lemma 2.4 of [12], this is always true for some i.r. Regard $\Omega$ as an analytic subspace of the unit interval; and define $\bar{\pi}(u)=\int \pi(d(x)) d \lambda^{u}(x) . \pi=\bar{\pi}$ a.e., so $\bar{\pi}$ can be used instead of $\pi$ to effect the decomposition. Then every $\bar{\pi}^{-1}(\{\omega\})$ is saturated with respect to the equivalence relation on $U_{G}$.

In the case $(S \times G, \mu \times h)$ of Example 1.11, the theorem gives a decomposition $\mu=\int \mu_{\omega} d \eta(\omega)$ so that a.a. $\lambda_{\omega}$ are product measures, $\left[\lambda_{\omega}\right]=\left[\mu_{\omega}\right.$ $\times h$ ]. Symmetry of a.a. of these measures implies a.a. $\mu_{\omega}$ are $G$-quasiinvariant [21, Corollary 2.10]. Thus we can derive the following corollary, due to Dang-Ngoc-Nghiem [4] and Kleppner [14]:

COROLlaRY 6.3. Let $(S, \mu)$ be an analytic quasi-invariant probability $G$-space 
for the separable locally compact group $G$ with Haar measure $h$. There is a standard probability space $(\Omega, \eta)$, a conull invariant Borel subset $S_{0} \subset S$, and a Borel surjection $\pi: S_{0} \rightarrow \Omega$ such that $\pi_{*} \mu=\eta$ and $\mu$ has on $S_{0}$ a $\pi$-decomposition $\mu=\int \mu_{\omega} d \eta(\omega)$ such that every $\left(S_{0}, \mu_{\omega}\right)$ is an ergodic $G$-space.

Proof. Let $E_{1}, E_{2}, \ldots$ be a generating algebra for $\Re(S \times G)$. Let $m \in[h]$ be a symmetric probability. Let $q=d m / d h, \Delta$ the modular homomorphism of $(\mu \times h, \mu)$. Let $\Omega_{1}=\left\{\omega \in \Omega: \int 1_{E_{i}}\left(s x, x^{-1}\right) d \mu_{\omega} \times m(s, x)\right.$ $=\int 1_{E_{i}}(s, x) \Delta(s, x)^{-1} q\left(x^{-1}\right) / q(x) d \mu_{\omega} \times m(s, x)$ for all $i \in \mathbf{N}$ and $\left\{I I_{1_{F}}^{\omega}: F\right.$ $\left.\left.=E_{1}, E_{2}, \ldots\right\}^{\prime}=\mathbf{C} I\right\}$. Let $\Omega_{0}$ be a relatively standard conull Borel subset of $\Omega_{1}$, let $S_{0}=\pi^{-1}\left(\Omega_{0}\right)$, and replace $\Omega$ by $\Omega_{0}$.

\section{REFERENCES}

1. A. Connes, Structure theory for type III factors, Proc. Internat. Congr. Math., vol. 2, Canadian Math. Congress, Vancouver, B. C., 1974, pp. 84-91.

2. _ A factor not anti-isomorphic to itself, Ann. of Math. 101 (1975), 536-554.

3. A. Connes and M. Takesaki, The flow of weights on factors of type III (preprint).

4. Dang-Ngoc-Nghiem, Décomposition et classification des systèmes dynamiques, Bull. Soc. Math. France 103 (1975), 149-175.

5. J. Dixmier, Les algèbres d'operateurs dans l'espace Hilbertien (Algèbres de von Neumann), Gauthier-Villars, Paris, 1957.

6. __ Les $C^{*}$-algèbres et leurs représentations, 2nd ed., Gauthier-Villars, Paris, 1969.

7. J. Feldman, P. Hahn and C. Moore, Orbit structure and countable sections for actions of continuous groups (preprint).

8. J. Feldman and C. Moore, Ergodic equivalence relations, cohomology, and von Neumann algebras. I, Trans. Amer. Math. Soc. 234 (1977), 289-324.

9. , Ergodic equivalence relations, cohomology, and von Neumann algebras. II, Trans. Amer. Math. Soc. 234 (1977), 325-359.

10. J. Glimm, Families of induced representations, Pacific J. Math. 12 (1962), 885-911.

11. P. Hahn, Haar measure and convolution algebras on ergodic groupoids, Thesis, Harvard Univ., Cambridge, Mass., 1975.

12. __ Haar measure for measure groupoids, Trans. Amer. Math. Soc. 242 (1978), 1-33.

13. M. Heins, Complex function theory, Academic Press, New York, 1968.

14. A. Kleppner, Ergodic decompositions of quasi-invariant positive forms, Technical Report 74-39, Univ. of Maryland, 1974.

15. W. Krieger, On constructing non-*-isomorphic hyperfinite factors of type III, J. Functional Analysis 6 (1970), 97-109.

16. G. Mackey, Imprimitivity for representations of locally compact groups, Proc. Nat. Acad. Sci. U.S.A. 35 (1949), 537-545.

17. _ Borel structures in groups and their duals, Trans. Amer. Math. Soc. 85 (1957), 134-165.

18. _ Ergodic theory, group theory, and differential geometry, Proc. Nat. Acad. Sci. U.S.A. 50 (1963), 1184-1191.

19. _ Ergodicity in the theory of group representations, Proc. Internat. Congr. Math., vol. 2, Canadian Math. Congress, Vancouver, B. C., 1974, pp. 401-405.

20. A. Ramsay, Virtual groups and group actions, Advances in Math. 6 (1971), 253-322.

21. __ Boolean duals of virtual groups, J. Functional Analysis 15 (1974), 56-101.

22. (1976), 17-48.

23. ___ Subobjects of virtual groups (preprint). 
24. B. Russo, Review of "Product croisé d'une C*-algèbre par un groupe d'automorphismes," Math. Reviews 39 \#3329 (1970), 606-607.

25. M. Samuelides and J.-L. Sauvageot, Algèbre de Krieger d'un système dynamique, C. R. Acad. Sci. Paris Sér. A-B 280 (1975), A709-A712.

26. J.-L. Sauvageot, Image d'un homomorphisme et flot des poids d'une relation d'equivalence mesurée, C. R. Acad. Sci. Paris Sér. A-B 282 (1976), A619-A621.

27. M. Takesaki, Tomita's theory of modular Hilbert algebras and its applications, Lecture Notes in Math., vol. 128, Springer-Verlag, Berlin and New York, 1970.

28. J. Westman, Harmonic analysis on groupoids, Pacific J. Math. 27 (1968), 621-632.

29. , Nontransitive groupoid algebras (preprint).

30. __ Ergodic groupoid algebras and their representations (preprint).

31. G. Zeller-Meier, Produit croisé d'une $C^{*}$-algèbre par un groupe d'automorphismes, J. Math. Pures Appl. 47 (1968), 101-239.

Department of Mathematics, Untversity of California, Berkeley, California 94720

Current address: 10 Soldiers Field Park 10C, Boston, Massachusetts 02163 